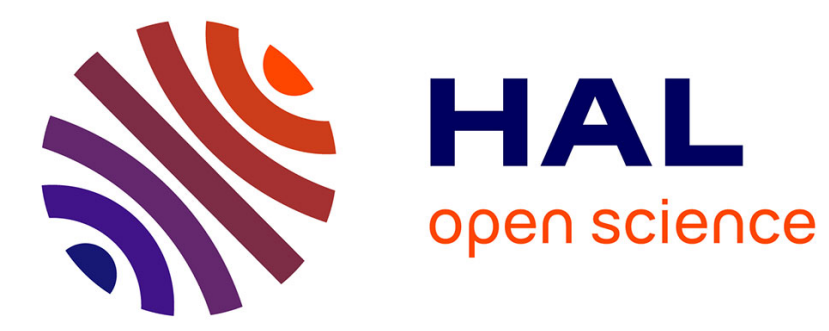

\title{
Wind Farm Portfolio Optimization under Network Capacity Constraints
}

\author{
Hélène Le Cadre, Anthony Papavasiliou, Yves Smeers
}

\section{To cite this version:}

Hélène Le Cadre, Anthony Papavasiliou, Yves Smeers. Wind Farm Portfolio Optimization under Network Capacity Constraints. European Journal of Operational Research, 2015, 247 (2), pp.560-574. 10.1016/j.ejor.2015.05.080 . hal-01007992v4

\section{HAL Id: hal-01007992 https://hal.science/hal-01007992v4}

Submitted on 4 Jun 2015

HAL is a multi-disciplinary open access archive for the deposit and dissemination of scientific research documents, whether they are published or not. The documents may come from teaching and research institutions in France or abroad, or from public or private research centers.
L'archive ouverte pluridisciplinaire HAL, est destinée au dépôt et à la diffusion de documents scientifiques de niveau recherche, publiés ou non, émanant des établissements d'enseignement et de recherche français ou étrangers, des laboratoires publics ou privés. 


\title{
Wind Farm Portfolio Optimization under Network Capacity Constraints*
}

\author{
Hélène Le Cadre ${ }^{\dagger} \quad$ Anthony Papavasiliou $\quad$ Yves Smeers $^{\ddagger}$
}

\begin{abstract}
In this article, we provide a new methodology for optimizing a portfolio of wind farms within a market environment, for two Market Designs (exogenous prices and endogenous prices). Our model is built on an agent based representation of suppliers and generators interacting in a certain number of geographic demand markets, organized as two tiered systems. Assuming rational expectation of the agents with respect to the outcome of the real-time market, suppliers take forward positions, which act as signals in the day-ahead market, to compensate for the uncertainty associated with supply and demand. Then, generators optimize their bilateral trades with the generators in the other markets. The Nash Equilibria resulting from this Signaling Game are characterized using Game Theory. The Markowitz Frontier, containing the set of efficient wind farm portfolios, is derived theoretically as a function of the number of wind farms and of their concentration. Finally, using a case study of France, Germany and Belgium, we simulate the Markowitz Frontier contour in the expected cost-risk plane.
\end{abstract}

Keywords: Game Theory; Market Design; Renewable Capacity Investment; Markowitz Frontier.

\section{Introduction}

Market Coupling is the most advanced Market Design in the restructuring of the European (EU) electricity market. Market Coupling is the current outcome of a long and possibly never ending process towards an integrated electricity market [8]. The path towards Market Coupling developed from an initially

*Acknowledgement: The authors would like to thank the three anonymous referees and the Editor for their helpful comments which have helped them to greatly improve their work. The second author acknowledges the support of the GDF Suez Chair in Energy Economics and Management of Energy Risk.

${ }^{\dagger}$ MINES ParisTech, PSL Research University, CMA - Center for Applied Mathematics, CS 10207 rue Claude Daunesse, 06904 Sophia Antipolis Cedex, France e-mail: helene.le_cadre@mines-paristech.fr

$\ddagger$ CORE, Center for Operations Research and Econometrics, Catholic University of Louvain, Belgium e-mails:\{anthony.papavasiliou,yves.smeers\}@uclouvain.be 
very decentralized view of an electricity market that only cared for access to the grid; development towards centralization then came as one progressively became aware of the very particular need for coordination required by electricity. Market Coupling takes some of these requirements into account but still remains far from the central organization that drives the United States (US) Independent System Operator (ISO) system. Market Coupling indeed relies on a separation of the energy market (organized by Power Exchanges) and transmission services (congestion and grid support services) operated by Transmission System Operators (TSOs). As several other electricity markets, Market Coupling is a two tiered system with a day-ahead market followed by a real-time market or, more exactly, a balancing system. The system is zonal in the sense that the European (EU) market is decomposed into zones (often, but not always, corresponding to countries) where Kirchhoff's laws are ignored and it is assumed that there is no congestion. Power Exchanges operate in national markets and a special supra national organization clears the global energy market on the basis of the bids submitted to the national Power Exchange and a representation of the grid coming from the TSOs [41.

Our work can be related to two streams of previous work. The first stream of literature is that related to the application of stochastic programming models to the electricity markets. Because events can always occur between day ahead and real time, the TSO can encounter in real time network difficulties that have not been anticipated in the day ahead. This phenomenon is enhanced in Market Coupling because market clearing in the day ahead uses an imperfect representation of the grid. In their paper, Smeers et al. 30 assessed the amount of counter trading that might arise in real time as a result of using an approximate description of the grid in the day ahead. This problem takes a further dimension with wind penetration as wind forecast remains imperfect and any market clearing based on such forecasts in the day ahead is bound to be in imbalance in real time. The issue was examined in Oggioni et al. 24] under strong assumptions of perfect coordination among TSOs to organize counter trading. In both cases, the work compared the result of a stylized representation of Market Coupling with an equally stylized representation of nodal pricing. Our second stream for inspiration comes from the computer systems literature [21. In their model, Nair et al. explicitly characterized the impact of growing wind power penetration on electricity market design by optimizing the placement of an intermediate market between the day ahead and real time, assuming supply and demand balance each other [21. The originality of their model stemmed from its capability to derive closed form expressions for the optimal energy procurement strategy by making the analogy with a variant of the well-known newsvendor problem.

Market Coupling is considered as a major achievement on the path to internal energy market; its progress has been discussed at length in EU circles such as the Regulatory Forum in Florence. Surprisingly the literature and in particular the formalized literature on the subject has remained relatively limited in contrast for instance with the extensive literature in the US nodal pricing system [23]. 
Our model extends the approach of Nair et al. by introducing competition among the geographic demand markets subject to Market Coupling and characterizing the optimal investment policy in renewable energy supply capacity, which is becoming a major consideration for utilities that are subject to aggressive renewable portfolio standards.

Accurate short-term forecasts of wind farm power output over the next few hours to days are important factors for secure and low cost operations of power systems with high wind power penetration [20, 25]. According to Girard et al. [7, it is difficult to quantify the economic benefit of improved forecasting. The recent literature dealing with the placement of wind turbines concludes that the aggregation of wind farms can produce significant effects in terms of variability and cost reduction 7 since forecast errors might compensate each other. Furthermore, a portfolio of wind farms is likely to give better results in terms of the trade-off between cost and profit and its variability than relying on a single wind farm 9 . Considering both problems of wind farm expansion and optimal wind farm portfolio generation, Girard et al. checked, using real data from Western Denmark, that power generator revenue is linear with respect to the wind farm capacity factor and that the accuracy of short-term wind power forecasts has only a very small impact on revenue. However, as the authors explained, their results do not quantify the benefit of predictability from a global system point of view. Adopting a more systemic approach, Green showed, using 18 years of hourly wind speed data coming from 120 sites around Great Britain, that careful market analysis is needed if investors are to build optimal portfolios of wind stations 9 . Baringo and Conejo already made the link, dealing with the optimization of a strategic wind power investor who sells wind power in a two tiered market (including a day-ahead and a balancing market) [1].

The main contribution of our work is an analytical treatment of the strategic positioning of suppliers in two tiered electricity markets, where real-time conditions are dictated by the uncertainty of renewable energy supply. A byproduct of this analysis is the characterization of the optimal investment policy in renewable energy supply capacity, for suppliers acting as investors in wind power.

According to the literature mentioned above, a careful (simplified) modeling of Market Coupling is the most crucial modeling aspect. We consider a certain number of geographic demand markets, described in Section 2 . Since we want to characterize the agents' general behaviors, we do not consider explicitly Kirchhoff's laws and aggregate the supply and the demand at the market level. The originality of our approach relies on its capability to cope with competition among suppliers that are operating in spatially separated electricity markets, which was ignored in the previously cited models. After having defined the agents' roles in Subsection 2.1, we assume that a Signaling Game occurs over each geographic demand market in the day ahead. Its timing is described in Subsection 2.2 The link between day-ahead and real-time markets is guaranteed by the existence of a forward position in the day-ahead energy market that compensates for the uncertainty of supply and demand in real time. The Game is solved for two Market Designs: two tiered with exogenous prices in Section 3 
and two tiered with endogenous prices in Section 4 . Efficient wind farm portfolios are then characterized on the basis of the Markowitz Frontier definition. Its exploration is detailed in Section 5. Contrary to traditional approaches that ignore the occurrence of rare events when performing risk minimization, the Markowitz Frontier is defined in a context of rare events, guaranteeing the robustness of the wind farm distribution. Illustrations based on real wind speed and energy consumption data for France, Germany and Belgium are provided in Section 6 .

\section{Specific notation and modeling assumptions}

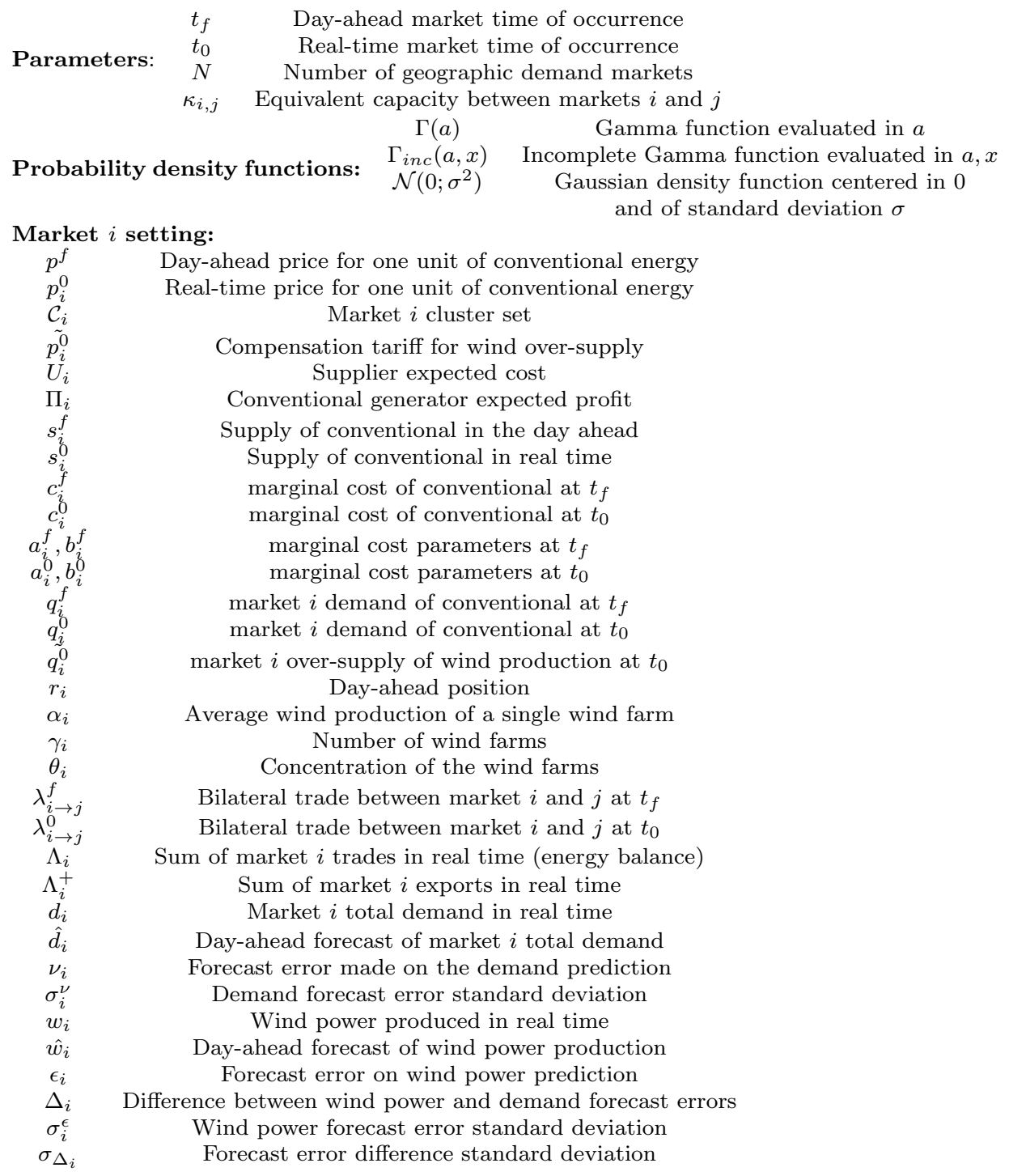




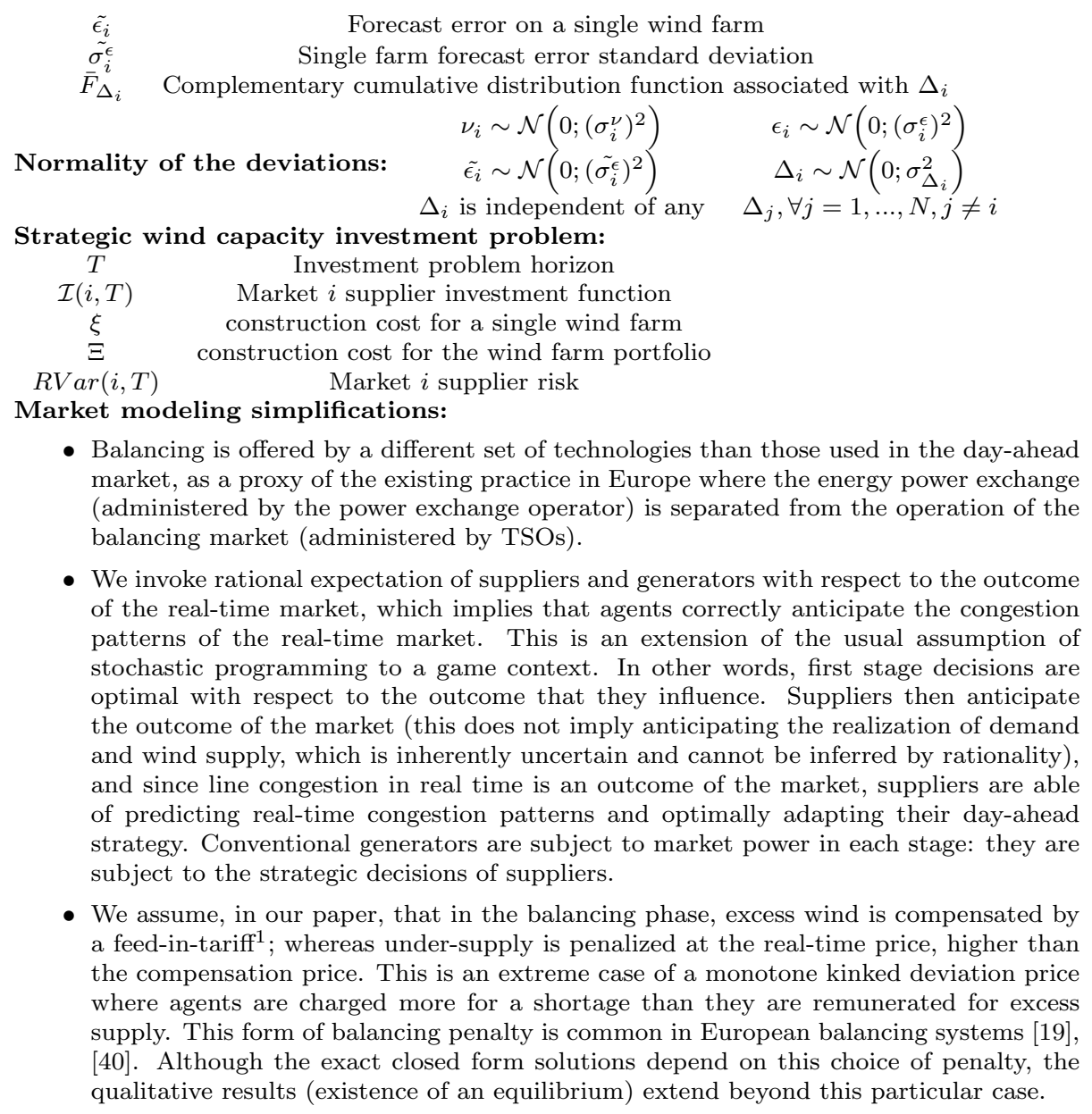

\section{The market model}

We consider suppliers (distributors or utility companies) with long term contracts for renewable energy. Given such a long term contract, the suppliers participate in a two tiered market for conventional energy production. It consists of a day-ahead market occurring at $t_{f}>0$ and of a real-time market, occurring at $t_{0}>t_{f}$; meaning that $t_{0}$ occurs after $t_{f}$. It is worth mentioning that we do not address bilateral forward contracting but adopt a standard assumption in the literature focusing on two-settlement systems [1, 19.

In the European Union (EU), the real-time markets introduced in this article can be assimilated to the EU balancing system [7, [14] where positive and negative deviations are treated asymmetrically. Positive deviations are reimbursed on the basis of a fixed tariff by excess unit, supposed smaller than the real-time

\footnotetext{
${ }^{1}$ The Feed-in-tariff, http://www.uswitch.com/solar-panels/guides/feed-in-tariff/
} 
price (this can be compared with the so called feed-in-tariff), whereas negative deviations pay the real-time price as in Nair et al. model [21. This reflects an uninstructed deviation penalty for discouraging an unscheduled over-supply of renewable power in real time, and is consistent with existing market practices. Asymmetric settlements of real-time deviations have also been modeled in the literature [19, 21], 27].

Our model is based on the assumption that competition is pure and perfect within each geographic demand market in the day ahead and in real time. That is why we assume throughout the article that offer equals demand (and also, price equals marginal cost). Furthermore, we invoke rational expectation in order to argue that agents correctly anticipate the congestion of a certain set of lines.

Inside the economic system formed by the power markets, bilateral trades occur among the markets. We let $\lambda_{i \rightarrow j}^{f}\left(\right.$ resp. $\lambda_{i \rightarrow j}^{0}$ ) be the bilaterally traded flow of energy between market $i$ and market $j$ in the day-ahead market (resp. real-time market). A negative sign indicates an import from $j$ to $i$, whereas a positive sign indicates an export from $i$ to $j$. Throughout the article, we will use the simplifying notations: $\Lambda_{i} \triangleq \sum_{j \neq i} \lambda_{i \rightarrow j}^{0}, \Lambda_{i}^{+} \triangleq \sum_{j \neq i}\left(\lambda_{i \rightarrow j}^{0}\right)_{+}$and the following conventions: $(x)_{+} \triangleq \max \{x ; 0\}$ and $(x)_{-} \triangleq \max \{-x ; 0\}, \forall x \in \mathbb{R}$.

Market Coupling clears energy and transmission in the day ahead with an "implicit auction" of transmission capacity [10, [22, and is followed by intraday trading and real-time balancing. Commonly, markets having smaller prices export to markets having higher prices, until a common price is reached or congestion occurs due to limited Available Transmission Capacities at the interconnections [6], 13, [30. Congestion management remains a controversial issue in the restructured European electricity sector. Congestion occurs when the infrastructure constrains transactions, thereby influencing the long term evolution of generation and consumption $[6]$.

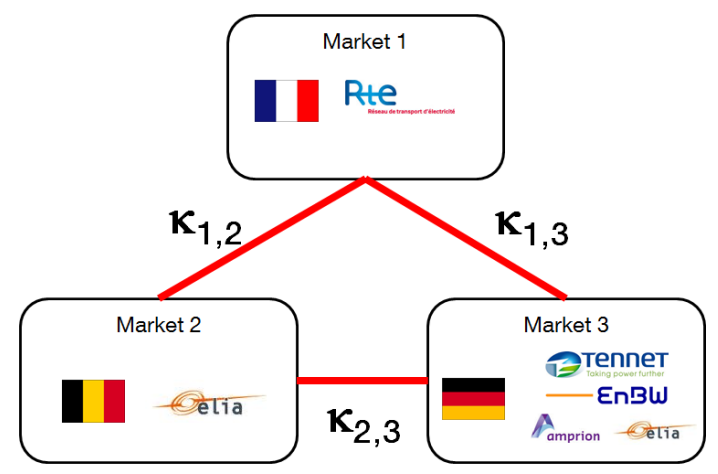

Figure 1: Stylized representation of three split geographic demand markets: France, Belgium and Germany, with their associated TSOs.

Our network model is based on a virtual representation of the European area 
where each node coincides with a geographi ${ }^{2}$ demand market containing one or more TSOs and is fully interconnected with other geographic demand markets. We give an illustration of this representation in Figure 1 for three geographic demand markets $(N=3)$. Inside each demand market, the balancing markets (managed by the TSOs) are coupled whereas they are split with the $N-1$ other demand markets. We introduce the following definition: geographic demand markets are coupled in real time if, and only if, their real-time prices coincide i.e., no congestion occurs in real tim£ ${ }^{3}$.therwise, they are said to be split. We introduce the equivalent interconnection capacity between market $i$ and market $j: \kappa_{i, j} \in \mathbb{R}$ such that $\kappa_{i, j}<+\infty, i \neq j$. According to Hutcheon and Bialek's representation of the European system [11, for any $i, j=1, \ldots, N, i \neq j, \kappa_{i, j}$ can be either positive or negative; furthermore, we have the relation: $\kappa_{i, j}=-\kappa_{j, i}$. We assume that all agents correctly anticipate the congestion of a certain set of lines leading to a virtual representation of the European area in $N$ split demand markets. Such an anticipation has already been done by Borenstein et al. in [4] for two geographic markets sharing a constrained transmission line. As a by-product of congestion anticipation, for any couple of demand markets, realtime trade equals equivalent interconnection capacity minus day-ahead trade i.e., $\lambda_{i \rightarrow j}^{0}=\kappa_{i, j}-\lambda_{i \rightarrow j}^{f}, \forall i, j=1, \ldots, N, i \neq j$.

\subsection{The agents}

There is a certain number of geographic demand markets, each characterized by a price insensitive ${ }^{4}$ and random demand. Only forecasts of the demand are available in the day ahead and the true values of the demand (i.e., its realizations) are revealed in real time. Similarly, wind generation in each geographic market is price insensitive and random: only forecasts are available in the day ahead and the true values (i.e., the random variables realizations) are revealed in real time. We now describe the different categories of agents interacting in each demand market.

- Suppliers (distributors or utility companies) deliver energy to consumers characterized by their aggregated demand. They are price takers in the first Market Design (MD 1) detailed in Section 3. In contrast with standard assumptions, they are not price takers in the second Market Design (MD 2), described in Section 4. In this latter Market Design, they are aware that their decisions modify prices and take that knowledge into account to minimize their procurement cost. Since consumers are exposed to a retail price that is unrelated to wholesale market conditions, we assume an inelastic consumer demand whose real-time level is uncertain in the day-ahead time frame. Additionally, we assume that the suppliers are

\footnotetext{
${ }^{2}$ The term "geographic" will be understood in the rest of the article.

${ }^{3}$ The case of uncongested markets in real time is studied in [15].

${ }^{4}$ We do not consider demand side management in the present article. Models dealing with decentralized demand response integration through distributed learning approaches can be found in [14]. In [16], the end user's demand is price responsive and storage is possible either at the end users' level, or at the microgrid aggregator level.
} 
driven by renewable portfolio standards to invest in renewable capacity. Suppliers have to decide how to compose an optimal wind farm portfolio for participating in the electricity market.

- Conventional energy generators are characterized by their supply function. There is conventional generation in each market. Marginal costs are higher in real time than in the day ahead for a given output level. We assume that generators do not exercise market power, and suppliers buy electricity at marginal cost.

\subsection{A Signaling Game}

We make the assumption that a clearing price is reached at $t_{f}$. Because the transfers are limited by the equivalent interconnection capacity, it will be harder to align the market prices at $t_{0}$.

We now describe the optimization program faced by each market. Over each market $i$, at time instant $t_{f}$ :

(i) The suppliers optimize independently and simultaneously their purchase of conventional energy so as to minimize their expected cost while ensuring that the total purchased quantity satisfies the residual demand.

(ii) Anticipating what will happen in the real time market i.e., at time instant $t_{0}$, the conventional generators optimize independently and simultaneously the bilateral trades with the other markets so as to maximize their expected profit under equivalent capacity constraints.

Under congestion anticipation of all the equivalent interconnection capacities, the trades at $t_{f}$ are linked to the optimal trades at $t_{0}$ according to the relation: $\lambda_{i \rightarrow j}^{f}=\kappa_{i, j}-\lambda_{i \rightarrow j}^{0}, \forall i, j, i \neq j$. The distributed optimization of the program described above gives rise to a game belonging to the family of Signaling Games 31. Here the signal is based on the suppliers' forward positions which can be expressed as mappings of the suppliers' wind and demand forecasts of the underlying state of the system (unknown in the day ahead): $\left(w_{i}, d_{i}\right)_{i=1, \ldots, N}$.

The bilevel game described above is solved by backward induction: we start by optimizing the generator's bilateral trades, assuming that the suppliers' forward positions are fixed. Then, the supplier's expected cost are optimized by derivating their utility with respect to their purchase of conventional energy.

\subsection{Description of the markets}

Market $i$ is defined by:

- $d_{i}$, the end users' total demand of energy at time $t_{0}$. It satisfies the relation: $d_{i}=\hat{d}_{i}-\nu_{i}$ where $\hat{d}_{i}$ is the forecast made at $t_{f}$ of the end users' total demand of energy at $t_{0} . \nu_{i}$ is a random variable, representing the forecast error made on the demand prediction, and distributed according 
to a Gaussian density function centered in 0 and of standard deviation $\sigma_{i}^{\nu}$ : $\nu_{i} \sim \mathcal{N}\left(0 ;\left(\sigma_{i}^{\nu}\right)^{2}\right)$.

- $w_{i}$, the energy produced at time $t_{0}$ by the market renewable energy generators. It satisfies the relation: $w_{i}=\hat{w}_{i}-\epsilon_{i}$ where $\hat{w}_{i}$ is the forecast made at $t_{f}$ of the quantity of renewable energy that market $i$ generator will produce at $t_{0} . \epsilon_{i}$ is a random variable, representing the forecast error made on the prediction of the renewable production, distributed according to a Gaussian density function centered in 0 and of standard deviation $\sigma_{i}^{\epsilon}$ : $\epsilon_{i} \sim \mathcal{N}\left(0 ;\left(\sigma_{i}^{\epsilon}\right)^{2}\right)$. The forecast error on the production of a single wind farm will be denoted $\tilde{\epsilon}_{i}$. Being consistent with the assumption made on $\epsilon_{i}$ generation, it is distributed according to a Gaussian density function ${ }^{5}$ centered in 0 and of standard deviation $\tilde{\sigma}_{i}^{\epsilon}: \tilde{\epsilon}_{i} \sim \mathcal{N}\left(0 ;\left(\tilde{\sigma}_{i}^{\epsilon}\right)^{2}\right)$. The relation between $\epsilon_{i}$ and $\tilde{\epsilon}_{i}$ will be discussed explicitly in Subsection 2.5

- The forecast error vector for wind production and demand: $\left(\begin{array}{ll}\tilde{\epsilon}_{i} & \nu_{i}\end{array}\right)^{T}\left[\begin{array}{l}6 \\ \text { is }\end{array}\right.$ also supposed to be a Gaussian random vector. According to Sinden [29], wind power output in the United Kingdom (UK) has a weak positive correlation to current electricity demand patterns i.e., $\frac{\mathbb{E}\left[\tilde{\epsilon}_{i} \nu_{i}\right]}{\sigma_{i}^{\nu} \tilde{\sigma}_{i}^{\epsilon}}>0^{7}$. This implies that $\Delta_{i} \triangleq \epsilon_{i}-\nu_{i}$, which is the difference between renewable production and demand forecast errors, is distributed according to a Gaussian distribution function centered in 0 and of variance $\sigma_{\Delta_{i}}^{2}=\left(\sigma_{i}^{\epsilon}\right)^{2}-2 \mathbb{E}\left[\epsilon_{i} \nu_{i}\right]+\left(\sigma_{i}^{\nu}\right)^{2}$. In the rest of the article, we will let: $\Delta_{i} \sim f_{\Delta_{i}} \equiv \mathcal{N}\left(0 ; \sigma_{\Delta_{i}}^{2}\right) ; \bar{F}_{\Delta_{i}}$ will represent the associated complementary cumulative distribution function. $\Delta_{i}$ is supposed to be independent of any $\Delta_{j}, \forall j=1, \ldots, N, j \neq i$ i.e., the prediction errors made on one geographic market are independent of the ones made on the other geographic markets.

- $s_{i}^{f}$ (resp. $s_{i}^{0}$ ) market $i$ supply of conventional energy in day-ahead (resp. real-time) markets.

- $c_{i}^{f}\left(s_{i}^{f}\right)=a_{i}^{f}+b_{i}^{f} s_{i}^{f}\left(\right.$ resp. $\left.c_{i}^{0}\left(s_{i}^{0}\right)=a_{i}^{0}+b_{i}^{0} s_{i}^{0}\right)$ the marginal cost function of conventional energy produced by market $i$ and purchased at $t_{f}$ (resp. $t_{0}$ ), with $a_{i}^{0}>a_{i}^{f}>0$ and $b_{i}^{0}>b_{i}^{f}>0$ guaranteeing that the marginal cost on the real-time market remains larger than in the day-ahead market for a given output level. Indeed, balancing is often supplied from machines that are reserved on a long-term basis, and these are the older machines. The two supply curves are then different because they come from different machines. If so, the current formulation that separates

\footnotetext{
${ }^{5}$ Other density functions might be considered without adding any changes in the derived theoretical results except in the numerical illustrations where the Gaussian assumption greatly simplified the computations.

6.T stands for the transpose of the vector.

${ }^{7}$ Data analysis ran on 66 onshore weather recording sites for the period $1970-2003$ in the UK showed a correlation of 0.28 [29]. This is the value that we will use in the simulations.
} 
day-ahead and real-time machines, the latter having a higher marginal cost than the former, may be seen as a simplification made to preserve analytical tractability but is also in line with EU type systems where there is separated fleets of capacity for balancing and for the day-ahead market. Balancing capacity is contracted one year in advance [37, [38, 39], 40]. In addition, since the balancing market is pay-as-bid there is anyways no way of empirically verifying or refuting whether strong links between dayahead market and balancing system exist. Our modeling approach is an alternative that facilitates the development of our analysis and approximates non-convexities associated with real-time operations such as the wear and tear of machinery in emergency operation (emergency start up costs, wear due to emergency ramping, etc.).

- $q_{i}^{f}$ (resp. $q_{i}^{0}$ ) market $i$ demand of conventional energy in day-ahead (resp. real-time) markets.

The amounts of conventional energy purchased by market $i$ at $t_{f}$ and at $t_{0}$ are defined as follows: $q_{i}^{f}=\left(\hat{d}_{i}-\hat{w}_{i}+r_{i}\right)_{+}$and $q_{i}^{0}=\left(d_{i}-w_{i}-q_{i}^{f}\right)_{+}$where $r_{i}$ is a position in the day-ahead (lower cost) market. This day-ahead position is determined by the energy supplier in market $i$ for the consumers' demand $d_{i}$ to be satisfied at $t_{0}$ at the lowest possible cost; taking into account the uncertainty of supply at $t_{0}$. Market $i$ knows $\hat{d}_{i}$ and $\hat{w}_{i}$. Hence it is equivalent for the supplier to determine $q_{i}^{f}$ or $r_{i}$. The hypothesis that $q_{i}^{f}>0$ holds as long as the demand exceeds the average wind capacity 8 . In the rest of the article, we will assume that: $q_{i}^{f} \triangleq \hat{d}_{i}-\hat{w}_{i}+r_{i}$. The over-supply of wind production in real time is defined as: $\tilde{q_{i}^{0}}=\left(\Delta_{i}-r_{i}\right)_{-}$. Depending on the market mechanism, excess wind supply can be:

- Curtailed and compensated based on a feed-in-tariff. However, feed-intariffs are highly controversial and many countries wish that renewable energy generators directly participate to the market [26], 28]. We focus on this mechanism in this article.

- Bought by hydro-power generators and stored in their Energy Transfer by Pumping Stations. Hydro-power generators will re-inject extra supply later into the system in case of peak demand. This mechanism would require to introduce dynamicity in the agents' decisions and explicitly model the storage capabilities of the hydro-power generators. It is therefore out of the scope of this article.

- Re-injected into the system. If the ramp down of conventional generators (especially, gas and coal) is not committed in the day ahead (the start up costs being so high that the generators prefer producing power in excess

\footnotetext{
${ }^{8}$ It is sufficient to impose an upper-bound on the degree of wind penetration that we consider i.e., $\frac{\hat{d}_{i}+r_{i}}{\alpha_{i}}>\gamma_{i}$, to ensure that this inequality holds.
} 
than turning off their units), negative prices might appear. This mechanism is also out of the scope of this article because it would require to explicitly model the unit-commitment and the start-up cost of the conventional generators.

\subsection{Suppliers' expected cost and generators' expected prof- its}

We let $p^{f}$ be the day-ahead price, $p_{i}^{0}$ be market $i$ real-time price and $\tilde{p_{i}^{0}}<p_{i}^{0}$, the compensation price in case of excess wind. We define $U_{i}$, as the expected cost at $t_{f}$, that the supplier has to pay for its end user energy consumption. The supplier is penalized paying the real-time price per missing unit and is compensated on the basis of $\tilde{p_{i}^{0}}$ per wind unit in excess [27]:

$$
U_{i}=q_{i}^{f} p^{f}+\mathbb{E}\left[q_{i}^{0} p_{i}^{0}-\tilde{q}_{i}^{0} \tilde{p}_{i}^{0}\right]
$$

We let $\Pi_{i}$ be the expected profit at $t_{f}$ of market $i$ conventional energy generator. It is defined as the difference between the price paid by all the markets for the purchase of conventional energy and the cost of the energy. We assume that all the supply is sold at each time. Then:

$$
\begin{aligned}
\Pi_{i} & =s_{i}^{f} p^{f}-\int_{0}^{s_{i}^{f}} c_{i}^{f}(s) d s+\mathbb{E}\left[\sum_{j \neq i}\left(\lambda_{i \rightarrow j}^{0}\right)_{+} p_{j}^{0}\right]+\mathbb{E}\left[\left(s_{i}^{0}-\sum_{j \neq i}\left(\lambda_{i \rightarrow j}^{0}\right)_{+}\right) p_{i}^{0}\right] \\
& -\mathbb{E}\left[\int_{0}^{s_{i}^{0}} c_{i}^{0}(s) d s\right]
\end{aligned}
$$

\subsection{Renewable energy modeling}

The renewable wind energy production of market $i$ is a function of the number of wind farms and of their concentration which is characterized by their spatial distribution over market $i$ geographic area. To determine the renewable energy procurement for market $i$, we use the model of Nair et al. 21]. For market $i$, we introduce:

- $\alpha_{i}$, the average wind production of a single wind farm over the geographic area of market $i$.

- $\gamma_{i}$, the number of wind farms over market $i$ geographic area.

- $\theta_{i} \in\left[\frac{1}{2} ; 1\right]$ (resp. $\left.1-\theta_{i} \in\left[0 ; \frac{1}{2}\right]\right)$, a constant capturing the concentration (resp. the scattering) of the wind farm locations over market $i$ geographic area. The more (resp. the less) concentration, the more (resp. the less) correlation there is between the wind farm productions.

We suppose that, at $t_{f}, \alpha_{i}$ is the best forecast of wind energy procurement of a wind farm [21. Then: $\hat{w}_{i}\left(\gamma_{i}\right)=\alpha_{i} \gamma_{i}$. The forecast error will depend 
on the wind farm concentration too, and we choose the coefficient $\theta_{i}$ so that $\epsilon_{i}\left(\gamma_{i}\right)=\gamma_{i}^{\theta_{i}} \tilde{\epsilon}_{i}$ where $\tilde{\epsilon}_{i}$, as already introduced, represents the forecast error for the production of a single wind farm. We propose the following interpretation for the scaling of $\theta_{i}$ : If the wind farms are co-located they will all produce the same quantity of energy at the same time i.e., their productions are strongly correlated. This is the case when $\theta_{i}=1$. This implies in turn that: $\epsilon_{i}=\gamma_{i} \tilde{\epsilon}_{i}$ and that: $\hat{w}_{i}=w_{i}+\gamma_{i} \tilde{\epsilon}_{i}$. On the contrary, if they are spatially distributed so that their productions are independent from one another i.e., uncorrelated, and under the assumption that the forecast errors are distributed according to Gaussian distribution functions, the Central Limit Theorem tells us that: $\sigma_{i}^{\epsilon}=\sqrt{\gamma_{i}} \tilde{\sigma}_{i}^{\epsilon}$ 21. Therefore, the wind farm productions are independent from one another if, and only if, $\theta_{i}=\frac{1}{2}$. Note that in case of more general forecast error distribution functions, it can be interpreted as an approximation for $\gamma_{i}$ large enough. Finally, in case where $\left.\theta_{i} \in\right] \frac{1}{2} ; 1$ [, the wind farms are randomly located over the market geographic area and their spatial distribution is intermediate between perfect independence and co-location. With these notations, we obtain:

$$
\begin{aligned}
w_{i}\left(\gamma_{i}\right) & =\hat{w}_{i}\left(\gamma_{i}\right)-\epsilon_{i}\left(\gamma_{i}\right)=\alpha_{i} \gamma_{i}-\gamma_{i}^{\theta_{i}} \tilde{\epsilon}_{i} \\
\sigma_{i}^{\epsilon}\left(\gamma_{i}\right) & =\gamma_{i}^{\theta_{i}} \tilde{\sigma}_{i}^{\epsilon} \\
\epsilon_{i}\left(\gamma_{i}\right) & =\gamma_{i}^{\theta_{i}} \tilde{\epsilon}_{i}
\end{aligned}
$$

In the rest of the article, for the sake of simplicity, the dependence of $w_{i}, \sigma_{i}^{\epsilon}$ and $\epsilon_{i}$ on $\gamma_{i}$ will be omitted.

In the following sections, we solve the Signaling Game described in Subsection 2.2 for two Market Designs.

\section{MD 1: two tiered with exogenous prices}

In this first Market Design, the prices are supposed exogenous and such that:

$$
0<p^{f}<p_{i}^{0}, \forall i=1, \ldots, N
$$

Going backwards, we start with Step (ii) in the bilevel game described in Subsection 2.2. Derivating market $i$ generator's expected profit with respect to $\lambda_{i \rightarrow j}^{0}$ and assuming that $\lambda_{i \rightarrow j}^{0} \geq 0$, we obtain: $\frac{\partial \Pi_{i}}{\partial \lambda_{i \rightarrow j}^{0}}=-p^{f}+a_{i}^{f}+b_{i}^{f}\left(\hat{d}_{i}-\hat{w}_{i}+\right.$ $\left.r_{i}+\sum_{k} \kappa_{i, k}-\Lambda_{i}\right)+p_{j}^{0}-a_{i}^{0}-b_{i}^{0} \mathbb{E}\left[\left(\Delta_{i}-r_{i}\right)_{+}+\Lambda_{i}\right]$. We will now prove that market $i$ generator's optimal trades with the other markets is either zero or is reached at the saturation of the equivalent capacities.

Reasoning by contradiction, if there exists a $\lambda_{i \rightarrow j}^{0} \geq 0$ such that $\frac{\partial \Pi_{i}}{\partial \lambda_{i \rightarrow j}^{0}}=0$, this would mean that $p_{j}^{0}$ can be expressed exclusively as a function of market $i$ parameters. Suppose that there exists another market $k \neq j$ and $k \neq i$ such that $\lambda_{i \rightarrow k}^{0} \geq 0$, then necessarily, $p_{k}^{0}$ and $p_{j}^{0}$ should coincide. But, by assumption, realtime prices in different geographic demand markets do not coincide. Therefore, if there are more than two geographic demand markets, the maximum of $\Pi_{i}$ is reached at one boundary i.e., either $\lambda_{i \rightarrow j}^{0}=0$ or $\lambda_{i \rightarrow j}^{0}=\kappa_{i, j}$. 
We now turn to Step (i) in the bilevel game described in Subsection 2.2 Market $i$ supplier's expected cost takes the form:

$$
\begin{aligned}
U_{i} & =q_{i}^{f} p^{f}+\mathbb{E}\left[q_{i}^{0} p_{i}^{0}-\tilde{q_{i}^{0}} \tilde{p}_{i}^{0}\right] \\
& =\left(\hat{d}_{i}-\hat{w}_{i}+r_{i}\right) p^{f}+p_{i}^{0} \mathbb{E}\left[\left(\Delta_{i}-r_{i}\right)_{+}\right]-\tilde{p_{i}^{0}} \mathbb{E}\left[\left(\Delta_{i}-r_{i}\right)_{-}\right]
\end{aligned}
$$

We solve Step (i) of the optimization Program described in Subsection 2.2 In Step $(i)$, each market $i$ supplier determines independently and simultaneously the quantity of energy to purchase, $q_{i}^{f}$, or, equivalently, its day-ahead position, $r_{i}$, so as to minimize its expected procurement cost:

$$
\min _{r_{i}} \quad U_{i}
$$

The decision variable $r_{i}$ is in fact defined on a smaller space than the real coordinate space. Indeed, we need to have $r_{i} \geq 0$ since otherwise this means that a supplier could be short in the day ahead, something that one may find unrealistic given that conventional plants are more expensive in real time.

Derivating market $i$ expected cost with respect to $r_{i}$, we obtain: $\frac{\partial U_{i}}{\partial r_{i}}=$ $\left(p^{f}-\tilde{p_{i}^{0}}\right)-\left(p_{i}^{0}-\tilde{p_{i}^{0}}\right) \frac{\partial}{\partial r_{i}} \mathbb{E}\left[\Delta_{i}-r_{i} \mid\left\{\Delta_{i} \geq r_{i}\right\}\right]=\left(p^{f}-\tilde{p_{i}^{0}}\right)-\left(p_{i}^{0}-\tilde{p_{i}^{0}}\right) \bar{F}_{\Delta_{i}}\left(r_{i}\right)$. Then:

$$
\left.\frac{\partial U_{i}}{\partial r_{i}}\right|_{r_{i}=r_{i}^{*}}=0 \Leftrightarrow r_{i}^{*}=\bar{F}_{\Delta_{i}}^{-1}\left(\frac{p^{f}-\tilde{p_{i}^{0}}}{p_{i}^{0}-\tilde{p_{i}^{0}}}\right)
$$

Since $\frac{\partial^{2} U_{i}}{\partial r_{i}^{2}}=\left(p_{i}^{0}-\tilde{p_{i}^{0}}\right) f_{\Delta_{i}}\left(r_{i}\right)>0$, it coincides with a minimum for $U_{i}$. Furthermore, market $i$ 's optimal position being independent of the other markets' optimal positions, Equation (4) leads to a unique Nash Equilibrium for the market positions.

\section{MD 2: two tiered with endogenous prices}

In the following subsections, we derive analytically the endogenous prices in the day-ahead and in the real-time markets.

The global day-ahead market is characterized by the equilibrium between the supply and the demand: $q_{\text {tot }}^{f}(N)=\sum_{i=1, \ldots, N} q_{i}^{f}=\sum_{i=1, \ldots, N} s_{i}^{f}$ which is the global quantity of conventional energy exchanged on day-ahead markets. Furthermore, for any market $i$, we suppose, at $t_{0}$, that the difference between the supply and the demand for conventional energy in market $i$ coincides with the sum of bilateral trades with the other markets:

$$
\sum_{j=1, \ldots, N, j \neq i} \lambda_{i \rightarrow j}^{0}=s_{i}^{0}-q_{i}^{0} \Leftrightarrow \Lambda_{i}=s_{i}^{0}-q_{i}^{0}
$$


We make the assumption that the prices $p^{f}$ and $p_{i}^{0}$ paid by market $i$ suppliers for the energy purchased at $t_{f}$ and $t_{0}$ respectively equal the marginal costs. This assumption is justified in Subsection 2.3 by the decoupling of the cost functions at the day-ahead and real-time markets and the assumption of perfect competition which holds within each geographic demand market. In two-stage market models, represented by stochastic programming, such an assumption does not hold [27. There are two major reasons why we do not work with a two stage stochastic program at the generation side. One is the analytical character of our work. Our model assumes suppliers that obey a two-stage optimization paradigm with market power. Adding generators that also solve a two-stage optimization problem and finding the equilibrium between the suppliers and these generators is not amenable to an analytical solution. But, we also believe that this additional technical complexity would bring little in terms of realism in the EU context where the day-ahead market and the balancing are quite different systems that can in no way be modeled or approximated by two-stage stochastic program. The 2013 Survey on Ancillary Services Procurement and Electricity Balancing Market Design by ENTSO-E [36] shows that there is no relation in EU mind between what takes place in the energy market and in balancing. The maps contained in [36] also show that quite different systems can prevail in neighboring countries that are part of the Market Coupling, with the consequence that it is simply unrealistic to formulate the coupling of the day ahead and real time as a stochastic program. This separation is in fact our justification for our modeling of the system as two separate markets. It implies that: $p_{i}^{f}=c_{i}^{f}\left(s_{i}^{f}\right)$ and $p_{i}^{0}=c_{i}^{0}\left(s_{i}^{0}\right)$. Furthermore, we assume that a clearing price is reached at $t_{f}$ i.e., $p_{i}^{f}=p_{j}^{f} \triangleq p^{f}, \forall i, j=1, \ldots, N, i \neq j$ meaning that all the markets are integrated in a single one at that time. Because the transfers are limited by the available transmission capacities, it will be harder to align the market prices at $t_{0}$ : if the markets clear then $p_{i}^{0}=p_{j}^{0} \triangleq p^{0}, \forall i, j=1, \ldots, N, i \neq j$; otherwise there exists at least one market $i \in\{1, \ldots, N\}$ in which the supplier pays $p_{i}^{0} \neq p_{j}^{0}$ for $j \in$ $\{1, \ldots, N\}$ and $j \neq i$. This raises a combinatorial problem since all the possible combinations of split/coupled markets should be included in the model. But, the number of countries involved in the Central Western Europe area is currently limited to 6 (Netherlands, Belgium, France, Luxembourg, Germany and Italy since February 2015). Therefore, the number of combinations of split/coupled markets remains reasonable.

\subsection{Derivation of the coupling price}

We set: $A^{f} \triangleq \sum_{i=1, \ldots, N} \frac{a_{i}^{f}}{b_{i}^{f}}>0$ and $B^{f} \triangleq \sum_{i=1, \ldots, N} \frac{1}{b_{i}^{f}}>0$.

Lemma 1. The coupling price for the day-ahead market is: $p^{f}=\frac{\sum_{i=1, \ldots, N} q_{i}^{f}+A^{f}}{B^{f}}$.

Proof of Lemma 1. Using the assumption of the supply and demand equi- 
librium guaranteed by the day-ahead market rules, we have:

$$
\begin{aligned}
q_{\text {tot }}^{f}(N) & =\sum_{i=1, \ldots, N} q_{i}^{f}=\sum_{i=1, \ldots, N} s_{i}^{f} \\
& =\sum_{i=1, \ldots, N} \frac{p_{i}^{f}-a_{i}^{f}}{b_{i}^{f}} \text { under the assumption that } p_{i}^{f}=c_{i}^{f} . \\
& =\sum_{i=1, \ldots, N} \frac{p^{f}-a_{i}^{f}}{b_{i}^{f}} \text { since the } N \text { markets are coupled at } t_{f} . \\
& =p^{f}\left(\sum_{i=1, \ldots, N} \frac{1}{b_{i}^{f}}\right)-\sum_{i=1, \ldots, N} \frac{a_{i}^{f}}{b_{i}^{f}}
\end{aligned}
$$

We infer from the following equations the day-ahead price on the coupling zone.

\subsection{Derivation of the split market prices}

We set: $A_{i}^{0} \triangleq \frac{a_{i}^{0}}{b_{i}^{0}}>0$ and $B_{i}^{0} \triangleq \frac{1}{b_{i}^{0}}>0$. As in the proof of Lemma 1 we infer the real-time price on the $i$-th split market:

Lemma 2. The $N$ markets being split in $N$ geographic areas, at time $t_{0}$, market $i$ price for the real-time market $i s: p_{i}^{0}=\frac{q_{i}^{0}+A_{i}^{0}+\left[\sum_{j=1, \ldots, N, j \neq i} \lambda_{i \rightarrow j}^{0}\right]}{B_{i}^{0}}$.

Proof of Lemma 2. Using the real-time market rules defined through Equation 5 , we have: $q_{i}^{0}=s_{i}^{0}-\sum_{j=1, \ldots, N, j \neq i} \lambda_{i \rightarrow j}^{0}$. This implies that: $q_{i}^{0}+\sum_{j=1, \ldots, N, j \neq i} \lambda_{i \rightarrow j}^{0}=$ $p_{i}^{0} \frac{1}{b_{i}^{0}}-\frac{a_{i}^{0}}{b_{i}^{0}}$ using the fact that $c_{i}^{0}=p_{i}^{0}$. Then: $p_{i}^{0}=\frac{q_{i}^{0}+\frac{a_{i}^{0}}{b_{i}^{0}}+\left[\sum_{j=1, \ldots, N, j \neq i} \lambda_{i \rightarrow j}^{0}\right]}{\frac{1}{b_{i}^{0}}}=$ $\frac{q_{i}^{0}+A_{i}^{0}+\left[\sum_{j=1, \ldots, N, j \neq i} \lambda_{i \rightarrow j}^{0}\right]}{B_{i}^{0}}$ by definition of $A_{i}^{0}$ and $B_{i}^{0}$.

Day-ahead and real-time prices being now determined analytically, we let $\mathbf{r}_{-\mathbf{i}}$ be a $N-1$ dimensional vector containing the day-ahead positions of all the suppliers except market $i$ supplier.

Proposition 3. The sum of the bilateral trades of market $i$ in the real-time market, $\Lambda_{i}$, can be expressed as a linear function in $r_{i}$ and $\mathbf{r}_{-\mathbf{i}}$.

Proof of Proposition 3. By definition: $q_{i}^{f}=-\sum_{j \neq i} \lambda_{i \rightarrow j}^{f}+s_{i}^{f}$ and $p^{f}=$ $a_{i}^{f}+b_{i}^{f} s_{i}^{f}$. This implies, in turn, that: $\sum_{j \neq i} \lambda_{i \rightarrow j}^{f}=q_{i}^{f}-s_{i}^{f}$ and $s_{i}^{f}=\frac{p^{f}-a_{i}^{f}}{b_{i}^{f}}$. This implies that: $\sum_{j \neq i} \lambda_{i \rightarrow j}^{f}=q_{i}^{f}-\frac{p^{f}-a_{i}^{f}}{b_{i}^{f}}=q_{i}^{f}-\frac{\sum_{j} q_{j}^{f}+A^{f}}{B^{f} b_{i}^{f}}+\frac{a_{i}^{f}}{b_{i}^{f}}$ using the 
definition of $p^{f}$ obtained in Lemma 1. Then, under congestion anticipation: $\sum_{j \neq i} \lambda_{i \rightarrow j}^{0}=\sum_{j \neq i} \kappa_{i, j}-\sum_{j \neq i} \lambda_{i \rightarrow j}^{f}=\sum_{j \neq i} \kappa_{i, j}-\left(q_{i}^{f}-\frac{\sum_{j} q_{j}^{f}+A^{f}}{B^{f} b_{i}^{f}}+\frac{a_{i}^{f}}{b_{i}^{f}}\right)=$ $\left(\frac{1}{B^{f} b_{i}^{f}}-1\right)\left(\hat{d}_{i}-\hat{w}_{i}+r_{i}\right)+\frac{1}{B^{f} b_{i}^{f}} \sum_{j \neq i}\left(\hat{d}_{j}-\hat{w}_{j}+r_{j}\right)+\sum_{j \neq i} \kappa_{i, j}-\frac{1}{b_{i}^{f}}\left(a_{i}^{f}-\frac{A^{f}}{B^{f}}\right)$.

This means that there exists a linear function $\varphi_{i}: \mathbb{R}_{+}^{N} \rightarrow \mathbb{R}$ such that $\Lambda_{i}=\varphi_{i}\left(r_{i}, \mathbf{r}_{-\mathbf{i}}\right)$.

As a corollary of Proposition 3, we obtain: $\frac{\partial \Lambda_{i}}{\partial r_{i}}=\frac{\partial}{\partial r_{i}}\left(\sum_{j \neq i} \lambda_{i \rightarrow j}^{0}\right)=\frac{1}{B^{f} b_{i}^{f}}-1$ and $\frac{\partial \Lambda_{i}}{\partial r_{j}}=\frac{1}{B^{f} b_{i}^{f}}, \forall j \neq i$.

\subsection{Solving the Signaling Game}

The Signaling Game assumes that the supplier substitutes the day-ahead and real-time prices with linear functions of $r_{i}$ and $\mathbf{r}_{-\mathbf{i}}$ (this was proved in Proposition 3), and optimizes with respect to $r_{i}$, given $\mathbf{r}_{-\mathbf{i}}$. Day-ahead positions and bilateral trades cannot be derived analytically since, as we will see, it is intractable to inverse the complementary cumulative distribution function $\bar{F}_{\Delta_{i}}$ in the fixed point equation defining the Nash Equilibrium in the day-ahead positions ${ }^{9}$. However, we provide conditions guaranteeing the existence and uniqueness of a Nash Equilibrium for the day-ahead positions and detail algorithmically how day-ahead positions and bilateral trades at the optimum should be computed.

\subsubsection{Optimization of the bilateral trades}

Assuming that the agents correctly anticipate the congestion of the lines, we observe that the supplies of conventional energy on day-ahead and real-time markets can be expressed as functions of the bilateral trades in real time: $s_{i}^{f}=$ $\sum_{j \neq i} \lambda_{i \rightarrow j}^{f}+q_{i}^{f}=\left(\sum_{j \neq i} \kappa_{i, j}-\Lambda_{i}\right)+\left(\hat{d}_{i}-\hat{w}_{i}+r_{i}\right)$ and $s_{i}^{0}=\sum_{j \neq i} \lambda_{i \rightarrow j}^{0}+q_{i}^{0}=$ $\Lambda_{i}+\left(\Delta_{i}-r_{i}\right)_{+}$.

Proposition 4. The optimal bilateral trade between market $i$ and any market $j \neq i$ can be expressed as a linear function in $r_{j}, \mathbf{r}_{-j},\left(\mathbb{E}\left[\left(\Delta_{k}-r_{k}\right)_{+}\right]\right)_{k \mid p_{i}^{0}<p_{k}^{0}}$ and $\mathbb{E}\left[\left(\Delta_{j}-r_{j}\right)_{+}\right]$.

Proof of Proposition 4 Substituting $s_{i}^{f}$ and $s_{i}^{0}$ described above in the con-

\footnotetext{
${ }^{9}$ Except for simple distribution functions such as the Uniform density function.
} 
ventional energy generators' expected profit given in Equation (2), we obtain:

$$
\begin{aligned}
\Pi_{i} & =\left(\hat{d}_{i}-\hat{w}_{i}+r_{i}+\sum_{j \neq i} \kappa_{i, j}-\Lambda_{i}\right) \frac{1}{B^{f}}\left(\sum_{j}\left(\hat{d}_{j}-\hat{w}_{j}+r_{j}\right)+A^{f}\right) \\
& -a_{i}^{f}\left(\hat{d}_{i}-\hat{w}_{i}+r_{i}+\sum_{j \neq i} \kappa_{i, j}-\Lambda_{i}\right)-\frac{b_{i}^{f}}{2}\left(\hat{d}_{i}-\hat{w}_{i}+r_{i}+\sum_{j \neq i} \kappa_{i, j}-\Lambda_{i}\right)^{2} \\
& +\sum_{j \neq i}\left(\lambda_{i \rightarrow j}^{0}\right)_{+} \mathbb{E}\left[\frac{\left(\Delta_{j}-r_{j}\right)_{+}+A_{j}^{0}+\Lambda_{j}}{B_{j}^{0}}\right]-\frac{b_{i}^{0}}{2} \mathbb{E}\left[\left(\left(\Delta_{i}-r_{i}\right)_{+}+\Lambda_{i}\right)^{2}\right] \\
& +\mathbb{E}\left[\left(\Delta_{i}-r_{i}\right)_{+} \frac{1}{B_{i}^{0}}\left(\left(\Delta_{i}-r_{i}\right)_{+}+A_{i}^{0}+\Lambda_{i}\right)\right]-a_{i}^{0} \mathbb{E}\left[\left(\Delta_{i}-r_{i}\right)_{+}+\Lambda_{i}\right] \\
& +\mathbb{E}\left[\left(\Lambda_{i}-\sum_{j \neq i}\left(\lambda_{i \rightarrow j}^{0}\right)_{+}\right) \frac{\left(\Delta_{i}-r_{i}\right)_{+}+A_{i}^{0}+\Lambda_{i}}{B_{i}^{0}}\right]
\end{aligned}
$$

We suppose that there exists a $j$ in $\{1, \ldots, N\}$ such that $\lambda_{i \rightarrow j}^{0}>0$ (meaning that market $i$ is exporting to market $j$ ). We compute the derivative of $\Pi_{i}$ with respect to $\lambda_{i \rightarrow j}^{0}$ :

$$
\begin{aligned}
\frac{\partial \Pi_{i}}{\partial \lambda_{i \rightarrow j}^{0}} & =-\underbrace{\frac{\sum_{k}\left(\hat{d}_{k}-\hat{w}_{k}+r_{k}\right)+A^{f}}{B^{f}}}_{p^{f}}+\underbrace{a_{i}^{f}+b_{i}^{f}\left(\sum_{k \neq i} \kappa_{i, k}-\Lambda_{i}+\hat{d}_{i}-\hat{w}_{i}+r_{i}\right)}_{c_{i}^{f}\left(s_{i}^{f}\right)} \\
& -\frac{1}{B_{j}^{0}} \lambda_{i \rightarrow j}^{0}+\mathbb{E}\left[p_{j}^{0}\right]+\frac{1}{B_{i}^{0}}\left(\Lambda_{i}-\sum_{k \neq i}\left(\lambda_{i \rightarrow k}\right)_{+}+\mathbb{E}\left[\left(\Delta_{i}-r_{i}\right)_{+}\right]\right)-a_{i}^{0} \\
& -b_{i}^{0} \mathbb{E}\left[\left(\Delta_{i}-r_{i}\right)_{+}+\Lambda_{i}\right]
\end{aligned}
$$

We observe that the first line of the above equation vanishes since, by assumption, $p^{f}=c_{i}^{f}\left(s_{i}^{f}\right)$. Furthermore, since $\mathbb{E}\left[p_{j}^{0}\right]=\frac{1}{B_{j}^{0}} \mathbb{E}\left[\left(\Delta_{j}-r_{j}\right)_{+}\right]+c_{j}^{0}\left(\Lambda_{j}\right)$ and after a few simplifications, we obtain: $\frac{\partial \Pi_{i}}{\partial \lambda_{i \rightarrow j}^{0}}=-\frac{1}{B_{j}^{0}} \lambda_{i \rightarrow j}^{0}+\frac{1}{B_{j}^{0}} \mathbb{E}\left[\left(\Delta_{j}-r_{j}\right)_{+}\right]+$ $c_{j}^{0}\left(\Lambda_{j}\right)-c_{i}^{0}\left(\Lambda_{i}^{+}\right)-a_{i}^{0}$. Then:

$$
\frac{\partial \prod_{i}}{\partial \lambda_{i \rightarrow j}^{0}}=0 \Leftrightarrow \lambda_{i \rightarrow j}^{0}=B_{j}^{0}\left[c_{j}^{0}\left(\Lambda_{j}\right)-c_{i}^{0}\left(\Lambda_{i}^{+}\right)\right]+\mathbb{E}\left[\left(\Delta_{j}-r_{j}\right)_{+}\right]
$$

Summing $\lambda_{i \rightarrow j}^{0}$ over all the $j$ such that $\lambda_{i \rightarrow j}^{0}>0$ i.e., $\left\{j \mid p_{i}^{0}<p_{j}^{0}\right\}$, we obtain:

$$
\Lambda_{i}^{+}=\sum_{j \mid p_{i}^{0}<p_{j}^{0}}\left(B_{j}^{0}\left[c_{j}^{0}\left(\Lambda_{j}\right)-c_{i}^{0}\left(\Lambda_{i}^{+}\right)\right]+\mathbb{E}\left[\left(\Delta_{j}-r_{j}\right)_{+}\right]\right)
$$


Separating the equation in $\Lambda_{i}^{+}$we obtain:

$$
\begin{aligned}
\Lambda_{i}^{+} & =\frac{1}{1+b_{i}^{0} \sum_{j \mid p_{i}^{0}<p_{j}^{0}} B_{j}^{0}}\left\{\sum_{j \mid p_{i}^{0}<p_{j}^{0}}\left(B_{j} c_{j}^{0}\left(\Lambda_{j}\right)+\mathbb{E}\left[\left(\Delta_{j}-r_{j}\right)_{+}\right]\right)\right. \\
& \left.-a_{i}^{0} \sum_{j \mid p_{i}^{0}<p_{j}^{0}} B_{j}^{0}\right\}
\end{aligned}
$$

By substitution of Equation (7) in Equation (6), we obtain:

$$
\begin{aligned}
\lambda_{i \rightarrow j}^{0} & =B_{j}^{0}\left[c_{j}^{0}\left(\Lambda_{j}\right)-c_{i}^{0}\left(\frac { 1 } { 1 + b _ { i } ^ { 0 } \sum _ { k | p _ { i } ^ { 0 } < p _ { k } ^ { 0 } } B _ { k } ^ { 0 } } \left\{\sum _ { k | p _ { i } ^ { 0 } < p _ { k } ^ { 0 } } \left(B_{k} c_{k}^{0}\left(\Lambda_{k}\right)\right.\right.\right.\right. \\
& \left.\left.\left.\left.+\mathbb{E}\left[\left(\Delta_{k}-r_{k}\right)_{+}\right]\right)-a_{i}^{0} \sum_{k \mid p_{i}^{0}<p_{k}^{0}} B_{k}^{0}\right\}\right)\right]+\mathbb{E}\left[\left(\Delta_{j}-r_{j}\right)_{+}\right]
\end{aligned}
$$

This means that $\lambda_{i \rightarrow j}^{0}$ can be expressed exclusively as a linear function of $\Lambda_{j}$, $r_{j},\left(\mathbb{E}\left[\left(\Delta_{k}-r_{k}\right)_{+}\right]\right)_{k \mid p_{i}^{0}<p_{k}^{0}}$ and $\mathbb{E}\left[\left(\Delta_{j}-r_{j}\right)_{+}\right]$. But, we proved in Proposition 3 that there exists a linear function $\varphi_{j}: \mathbb{R}_{+}^{N} \rightarrow \mathbb{R}$ such that $\Lambda_{j}=\varphi_{j}\left(r_{j}, \mathbf{r}_{-\mathbf{j}}\right)$. As a result, at the optimum, $\lambda_{i \rightarrow j}^{0}$ can be expressed as a linear function in $r_{j}, \mathbf{r}_{-\mathbf{j}}$, $\left(\mathbb{E}\left[\left(\Delta_{k}-r_{k}\right)_{+}\right]\right)_{k \mid p_{i}^{0}<p_{k}^{0}}$ and $\mathbb{E}\left[\left(\Delta_{j}-r_{j}\right)_{+}\right]$.

\subsubsection{Minimization of the suppliers' expected cost}

We determined the analytical expressions of the endogenous coupling price for the integrated day-ahead market in Subsection 4.1 and of the endogenous prices for the split markets in real time in Subsection 4.2. Substituting these values in the suppliers' expected costs and using Proposition 3, each market $i$ supplier determines independently and simultaneously the quantity of energy to purchase, $q_{i}^{f}$, or, equivalently, its position, $r_{i}$, so as to minimize its expected procurement cost, as described in optimization Program 3 .

Market $i$ supplier determines the best answer, $r_{i}^{B A}\left(\mathbf{r}_{-\mathbf{i}}\right)$, which minimizes its expected procurement cost. The decentralized program output is a Nash Equilibrium, $\left(r_{i}^{*}\right)_{i=1, \ldots, N}$, defined by: $r_{i}^{*}=r_{i}^{B A}\left(\mathbf{r}_{-\mathbf{i}}^{*}\right), \forall i=1, \ldots, N$.

Proposition 5. If market $i$ energy balance is high enough (i.e., $\Lambda_{i}>\left(\tilde{p_{i}^{0}} B_{i}^{0}-\right.$ $\left.A_{i}^{0}\right)$ ), there exists a positive Nash Equilibrium solution of Program 3. Otherwise, the result still holds provided the standard deviation of $\Delta_{i}$ is smaller than $\frac{1}{\sqrt{2 \pi}}\left[\frac{\sum_{j \neq i} \lambda_{i \rightarrow j}^{0}-\left(\tilde{p}_{i}^{0} B_{i}^{0}-A_{i}^{0}\right)}{\frac{1}{2}\left(\frac{1}{B^{f} b_{i}^{f}}-3\right)-\frac{2 B_{i}^{0}}{B^{f}}}\right]$.

Proof of Proposition 5. It is provided in Appendix.

Using the methodology described in Proposition 5 proof, market $i$ determines the best answer: $r_{i}^{*}=r_{i}^{B A}\left(\mathbf{r}_{-i}^{*}\right)$ which minimizes its expected cost. Going a 
step further in the computations detailed in the proof of Proposition 5, we prove that this best answer is obtained as the solution of a fixed point equation: $\bar{F}_{\Delta_{i}}\left(r_{i}^{*}\right)=B_{i}^{0}\left\{A_{i}^{0}+\left(\frac{1}{B^{f} b_{i}^{f}}-1\right)\left(\hat{d}_{i}-\hat{w}_{i}+{\tilde{p_{i}^{0}}}_{B_{i}^{0}}+r_{i}^{*}\right)+\frac{1}{B^{f} b_{i}^{f}} \sum_{j \neq i}\left(\hat{d}_{j}-\right.\right.$ $\left.\left.\hat{w}_{j}+r_{j}^{*}\right)+\sum_{j \neq i} \kappa_{i, j}-\frac{1}{b_{i}^{f}}\left(a_{i}^{f}-\frac{A^{f}}{B^{f}}\right)-2 r_{i}^{*}\right\}^{-1}\left[\frac{\sum_{j}\left(\hat{d}_{j}-\hat{w}_{j}\right)+\sum_{j \neq i} r_{j}^{*}+r_{i}^{*}+A^{f}}{B^{f}}+\right.$ $\left.\frac{\hat{d}_{i}-\hat{w}_{i}+r_{i}^{*}}{B^{f}}-\tilde{p_{i}^{0}}+\left(\frac{1}{B^{f} b_{i}^{f}}+1\right) \frac{\sigma_{\Delta_{i}}}{B_{i}^{0} \sqrt{2 \pi}} \exp \left(-\frac{\left(r_{i}^{*}\right)^{2}}{2 \sigma_{\Delta_{i}}^{2}}\right)\right]$. This fixed point equation is solved simultaneously by all markets. Nash Equilibria are obtained at the intersections of the best answers.

To show uniqueness of the resulting Nash Equilibrium, we apply the contraction mapping approach. As shown in Bertsekas [2], it is sufficient to check that the Hessian of the expected cost functions fulfills the diagonal dominance condition i.e., $\sum_{j \neq i}\left|\frac{\partial^{2} U_{i}}{\partial r_{i} \partial r_{j}}\right|<\left|\frac{\partial^{2} U_{i}}{\partial r_{i}^{2}}\right|, \forall i=1, \ldots, N$. We show, below, that the uniqueness of the Nash Equilibrium heavily relies on the number of geographic demand markets:

Proposition 6. We assume that market $i$ energy balance is high enough (i.e., $\left.\Lambda_{i}>\left(\tilde{p_{i}^{0}} B_{i}^{0}-A_{i}^{0}\right)\right)$. If there are less or exactly three geographic demand markets then there exists a unique Nash Equilibrium solution of Program 3 .

Proof of Proposition 6. The assumption that $\Lambda_{i}>\left(\tilde{p_{i}^{0}} B_{i}^{0}-A_{i}^{0}\right)$ guarantees that Proposition 5 holds. Derivating first $U_{i}$ with respect to $r_{i}$ and then, a second time, with respect to $r_{j}, j \neq i$, we obtain: $\frac{\partial^{2} U_{i}}{\partial r_{i} \partial r_{j}}=\frac{1}{B^{f}}+\frac{b_{i}^{0}}{b_{i}^{f} B^{f}} \bar{F}_{\Delta_{i}}\left(r_{i}\right)>$ 0 . We remind that, by definition, $B^{f} b_{i}^{f}>1, \forall i=1, \ldots, N$. The assumption that $\Lambda_{i}>\left(\tilde{p}_{i}^{0} B_{i}^{0}-A_{i}^{0}\right)$ implies, according to Proposition $5 \frac{\partial^{2} U_{i}}{\partial r_{i}^{2}}-\sum_{j \neq i} \frac{\partial^{2} U_{i}}{\partial r_{i} \partial r_{j}}=$ $\frac{1}{B^{f}}(3-N)+b_{i}^{0}\left(3-\frac{N}{b_{i}^{f} B^{f}}\right) \bar{F}_{\Delta_{i}}\left(r_{i}\right)+\frac{\left.\Lambda_{i}-\left(\tilde{p_{i}^{0}} B_{i}^{0}-A_{i}^{0}\right)\right)}{B_{i}^{0}} f_{\Delta_{i}}\left(r_{i}\right)>\frac{1}{B^{f}}(3-N)+b_{i}^{0}(3-$

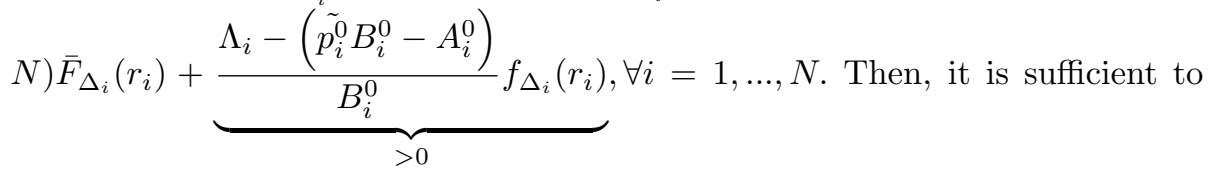
have $\frac{1}{B^{f}}(3-N)+b_{i}^{0}(3-N) \bar{F}_{\Delta_{i}}\left(r_{i}\right)>0 \Leftrightarrow(3-N) \underbrace{\left[\frac{1}{B^{f}}+b_{i}^{0} \bar{F}_{\Delta_{i}}\left(r_{i}\right)\right]}_{>0}>0$ to guarantee that the diagonal dominance condition holds. We conclude that if $N \leq 3$ then diagonal dominance is checked.

We have proved in Propositions 5 and 6 that for less or exactly three geographic demand markets, there exist parameter values guaranteeing the existence of a unique Nash Equilibrium solution of the Signaling Game described in Section 2.2. There is no guarantee about the uniqueness of a Nash Equilibrium for $N>3$. From a policy perspective, the existence of multiple Nash Equilibria implies that the outcome of the Market Design cannot be predicted. Computationally, multiple Nash Equilibria imply that the output of the model may depend on the initial conditions of the algorithm. 


\section{Wind farm portfolio optimization under di- rect participation of wind generator to the market}

When wind farm investor participates directly in an electricity market i.e., without subsidy-based supporting schemes, it influences the day-ahead positions, by putting wind in the system. In the following section, excess wind is not reimbursed anymore, leading to $\tilde{p_{i}^{0}}=0, \forall i=1, \ldots, N$. We assume that the investment strategy in renewable capacity is defined over a finite horizon $0<T<+\infty$. In this setting, the Signaling Game described in Section 4.3 is repeated over a finite horizon $T$.

There can be significant year-to-year variations in wind conditions, which would have an impact on profitability, and these may differ between regions 9 . Furthermore, the higher the terrain complexity, the lower the wind predictability. We assume that each market $i$ is clusterized in a subset $\mathcal{C}_{i}$ of clusters where, over $c \in \mathcal{C}_{i}$, the estimated demand $\hat{d}_{i}(c)$ and the wind mean production $\alpha_{i}(c)$ are supposed constant and such that at each time period $t$ :

$$
\begin{aligned}
d_{i}(c, t) & =\hat{d}_{i}(c)-\nu_{i}(c, t) \\
w_{i}(c, t) & =\hat{w}_{i}(c)-\epsilon_{i}(c, t)
\end{aligned}
$$

We assume that the geographic market $i$ clusters are defined so that there is no correlation among the cluster forecast errors because correlation among wind farms decreases with the distance [7] but that, inside each cluster, there remains a positive correlation between the wind production and the demand forecast error. Making the parallel with the previous notations, we have: $d_{i}(t)=$ $\sum_{c \in \mathcal{C}_{i}} d_{i}(c, t)$ and $\alpha_{i}=\sum_{c \in \mathcal{C}_{i}} \alpha_{i}(c)$. Clustering may be performed through one of the automatic partitioning algorithms used in Machine Learning [12], as illustrated for Germany in Section 6 .

We let $\Delta_{i}(c, t)$ be the difference between the wind power supply and the demand forecast errors in market $i$ cluster $c$, at time period $t$. Transposing Subsection 2.3 assumptions to a finer scale (i.e., clusters instead of geographic markets), we assume that $\left(\Delta_{i}(c, t)\right)_{c \in \mathcal{C}_{i}}$ is distributed according to a $\left|\mathcal{C}_{i}\right|$-dimensional Gaussian density function centered in the zero $\left|\mathcal{C}_{i}\right|$-dimensional vector and with a diagonal variance-covariance matrix having on its principal diagonal all the variances $\left(\sigma_{\Delta_{i}}(c)\right)^{2}, \forall c \in \mathcal{C}_{i}$ and zeros everywhere else since the covariance between any $\Delta_{i}(c, t), \Delta_{i}\left(c^{\prime}, t\right), \forall c, c^{\prime} \in \mathcal{C}_{i}, c \neq c^{\prime}$ vanishes. Furthermore, over each cluster $c \in \mathcal{C}_{i}$, the wind production and the demand forecast errors are correlated due to the assumption that $\left(\tilde{\epsilon_{i}}(c, t) \quad \nu_{i}(c, t)\right)^{T}$ is a Gaussian random vector centered in $\left(\begin{array}{l}0 \\ 0\end{array}\right)$ and of variance-covariance matrix 


$$
\left(\begin{array}{cc}
\left(\tilde{\sigma}_{i}^{\epsilon}(c)\right)^{2} & \mathbb{E}\left[\tilde{\epsilon}_{i}(c, t) \nu_{i}(c, t)\right] \\
\mathbb{E}\left[\tilde{\epsilon}_{i}(c, t) \nu_{i}(c, t)\right] & \left(\sigma_{i}^{\nu}(c)\right)^{2}
\end{array}\right)
$$

Modern Portfolio Theory is an alternative to the traditional method of analyzing each investment's individual merits. When investors look at each investment's individual merits, they are analyzing one investment without worrying about the way the different investments will perform relative to each other. On the other hand, Modern Portfolio Theory places a large emphasis on the correlation between the investments. Markowitz defines as efficient the portfolios which are characterized by a maximum expected revenue for a fixed risk (or, equivalently, for a minimum risk for a fixed expected revenue) [17. Risk and volatility are treated as the same thing: Markowitz uses risk as a measurement of the likelihood that an investment still goes up and down in value, and how often and by how much. The theory assumes that investors prefer to minimize risk. The Efficient Frontier, also called the Markowitz Frontier (MF), is then defined as the set of all the portfolios which are efficient. In this article, the supplier applies Modern Portfolio Theory to determine the wind farm portfolio that minimizes its expected investment while minimizing its risk conditionally to the occurrence of rare events 18. caused by discrepancies between forecasts and realizations. Note that since wind generator directly participates to the market and excess wind is curtailed, only under-supply is considered as a rare event.

In the numerical illustrations we will test two assumptions on the construction cost function for the wind farm portfolio on the cluster $c \in \mathcal{C}_{i}$ : either it is linear in the number of wind farms: $\Xi\left(\gamma_{i}(c)\right)=\xi \gamma_{i}(c)$ or it is quadratic: $\Xi\left(\gamma_{i}(c)\right)=\xi \gamma_{i}(c)^{2}$ with $\xi>0$ representing the construction cost for a single wind farm.

\subsection{In the $\left(\gamma_{i}(c), \theta_{i}(c)\right)$ plane}

In this subsection, we characterize analytically the MF in the $\left(\gamma_{i}(c), \theta_{i}(c)\right)$ plane. Theorem 7, described below, states that the optimal concentration of the wind farms $\theta_{i}(c)$ in cluster $c \in \mathcal{C}_{i}$ is obtained as the unique output of an explicit function of the number of wind farms $\gamma_{i}(c)$, provided $\gamma_{i}(c)$ satisfies the conditions of the second and third equations introduced in the Theorem. This is a strong result as we prove that the form of this explicit function is identical for MD 1 and MD 2. It is worth mentioning that Theorem 7 does not define a unique optimal portfolio but, in fact, a multiplicity of solutions.

Theorem 7. Whatever the Market Design (i.e., two tiered with exogenous prices, two tiered with endogenous prices), the Markowitz Frontier in the 
$\left(\gamma_{i}(c), \theta_{i}(c)\right)$ plane is completely described by the following set of equations:

$$
\begin{aligned}
\theta_{i}(c)= & \frac{\mathbb{E}\left[\tilde{\epsilon}_{i}(c) \nu_{i}(c)\right]}{\left(\tilde{\sigma_{i}}{ }^{\epsilon}(c)\right)^{2}} \\
\frac{\mathbb{L} \gamma_{i}(c)}{\left(\tilde{\epsilon}_{i}(c) \nu_{i}(c)\right]} & \left.\gamma_{i}(c) \leq\left(\frac{\mathbb{E}\left[\tilde{\epsilon}_{i}(c) \nu_{i}(c)\right]}{\left(\tilde{\sigma}_{i}{ }^{\epsilon}(c)\right)^{2}}\right)^{2}(c)\right)^{2} \\
& \gamma_{i}(c) \in \mathbb{N}^{*}
\end{aligned}
$$

Proof of Theorem 7. When optimizing its wind farm portfolio, market $i$ supplier's problem is to determine the optimal number of wind farms $\gamma_{i}(c) \in \mathbb{N}^{*}$ and their concentration $\frac{1}{2} \leq \theta_{i}(c) \leq 1$, over each cluster $c \in \mathcal{C}_{i}$, such that its expected investment is minimal and the variance of its investment conditionally to the occurrence of rare events is minimal.

MD 1: Over each geographic demand market $i=1, \ldots, N$, the supplier investment function is defined as the sum of the cost resulting from its conventional energy demand, repeated $T$ times, and of the cost devoted to the construction of the wind farm portfolio:

$$
\begin{aligned}
\mathcal{I}(i, T) & =\sum_{t}\left(q_{i}^{f}(t) p^{f}(t)+q_{i}^{0}(t) p^{0}(t)\right)+\sum_{c \in \mathcal{C}_{i}} \Xi\left(\gamma_{i}(c)\right) \\
& =\sum_{t}\left(\sum_{c \in \mathcal{C}_{i}}\left(\hat{d}_{i}(c)-\alpha_{i}(c) \gamma_{i}(c)\right)+r_{i}(t)\right) p^{f}(t) \\
& +\sum_{t}\left[\left(\sum_{c \in \mathcal{C}_{i}}\left(\tilde{\epsilon}_{i}(c, t) \gamma_{i}(c)^{\theta_{i}(c)}-\nu_{i}(c, t)\right)-r_{i}(t)\right)_{+} p_{i}^{0}(t)\right]+\sum_{c \in \mathcal{C}_{i}} \Xi\left(\gamma_{i}(c)\right) \\
& =\sum_{t}\left(\sum_{c \in \mathcal{C}_{i}}\left(\hat{d}_{i}(c)-\alpha_{i}(c) \gamma_{i}(c)\right)+r_{i}(t)\right) p^{f}(t)+\sum_{t}\left(\Delta_{i}(t)-r_{i}(t)\right)_{+} p_{i}^{0}(t) \\
& +\sum_{c \in \mathcal{C}_{i}} \Xi\left(\gamma_{i}(c)\right)
\end{aligned}
$$

where, we recall: $\Delta_{i}(t)=\sum_{c \in \mathcal{C}_{i}}\left(\epsilon_{i}(c, t)-\nu_{i}(c, t)\right)=\sum_{c \in \mathcal{C}_{i}}\left(\gamma_{i}(c)^{\theta_{i}(c)} \tilde{\epsilon}_{i}(c, t)-\right.$ $\left.\nu_{i}(c, t)\right)$. We obtain quite easily the analytical expression of the variance of $\Delta_{i}$ :

$$
\begin{aligned}
\sigma_{\Delta_{i}}^{2} & =\sum_{c \in \mathcal{C}_{i}} \sigma_{\Delta_{i}}^{2}(c)=\sum_{c \in \mathcal{C}_{i}} \operatorname{Var}\left(\epsilon_{i}(c)-\nu_{i}(c)\right) \\
& =\sum_{c \in \mathcal{C}_{i}} \operatorname{Var}\left(\gamma_{i}(c)^{\theta_{i}(c)} \tilde{\epsilon}_{i}(c)-\nu_{i}(c)\right) \\
& =\sum_{c \in \mathcal{C}_{i}}\left\{\left(\tilde{\sigma}_{i}{ }^{\epsilon}(c)\right)^{2} \gamma_{i}(c)^{2 \theta_{i}(c)}-2 \gamma_{i}(c)^{\theta_{i}(c)} \mathbb{E}\left[\tilde{\epsilon}_{i}(c) \nu_{i}(c)\right]+\sigma_{i}^{\nu}(c)^{2}\right\}(10)
\end{aligned}
$$


Then, we compute the supplier's risk i.e., the variance of its investment conditionally to the occurrence of rare events: $\operatorname{Var}\left(\mathcal{I}(i, T) \mid\left\{\Delta_{i}(t) \geq r_{i}(t)\right\}_{t}\right)=$ $\operatorname{Var}\left(\sum_{t} \Delta_{i}(t) p_{i}^{0}(t)-\sum_{t}\left(r_{i}(t) p_{i}^{0}(t)+\sum_{c \in \mathcal{C}_{i}}\left(\hat{d}_{i}(c)-\alpha_{i}(c) \gamma_{i}(c)+r_{i}(t)\right) p^{f}(t)\right)+\right.$ $\left.\sum_{c \in \mathcal{C}_{i}} \Xi\left(\gamma_{i}(c)\right)\right)$. We note that it is only the first term of this equation that depends on $\Delta_{i}(t)$. Using properties of the variance operator (i.e., variance of a constant equals zero, variance is a quadratic operator) and the fact that there is no dependence between two time consecutive forecast error differences, we infer that:

$$
\begin{aligned}
& \operatorname{Var}\left(\mathcal{I}(i, T) \mid\left\{\Delta_{i}(t) \geq r_{i}(t)\right\}_{t}\right)=\sum_{c \in \mathcal{C}_{i}} \sigma_{\Delta_{i}}^{2}(c) \sum_{t} p_{i}^{0}(t)^{2} \\
= & \sum_{c \in \mathcal{C}_{i}}\left[\left(\tilde{\sigma}_{i}^{\epsilon}(c)\right)^{2} \gamma_{i}(c)^{2 \theta_{i}(c)}-2 \gamma_{i}(c)^{\theta_{i}(c)} \mathbb{E}\left[\tilde{\epsilon}_{i}(c) \nu_{i}(c)\right]+\sigma_{i}^{\nu}(c)^{2}\right] \sum_{t} p_{i}^{0}(t)^{2}
\end{aligned}
$$

using Equation (10). In the rest of the article, we let:

$$
R \operatorname{Var}(i, T) \triangleq \operatorname{Var}\left(\mathcal{I}(i, T) \mid\left\{\Delta_{i}(t) \geq r_{i}(t)\right\}_{t}\right)
$$

be the risk of market $i$ supplier.

The expectation of the supplier's investment is:

$$
\begin{aligned}
\mathbb{E}[\mathcal{I}(i, T)] & =\sum_{t}\left(\sum_{c \in \mathcal{C}_{i}} \hat{d}_{i}(c)+\bar{F}_{\Delta_{i}}^{-1}\left(\frac{p^{f}(t)}{p_{i}^{0}(t)}\right)\right) p^{f}(t)-\sum_{t}\left(\sum_{c \in \mathcal{C}_{i}} \alpha_{i}(c) \gamma_{i}(c)\right) p^{f}(t) \\
& +\sum_{t} p_{i}^{0}(t) \mathbb{E}\left[\left(\Delta_{i}(t)-\bar{F}_{\Delta_{i}}^{-1}\left(\frac{p^{f}(t)}{p_{i}^{0}(t)}\right)\right)_{+}\right]+\sum_{c \in \mathcal{C}_{i}} \Xi\left(\gamma_{i}(c)\right)
\end{aligned}
$$

Derivating $R \operatorname{Var}(i, T)$ with respect to $\theta_{i}(c)$ and solving $\frac{\partial R \operatorname{Var}(i, T)}{\partial \theta_{i}(c)}=0$,

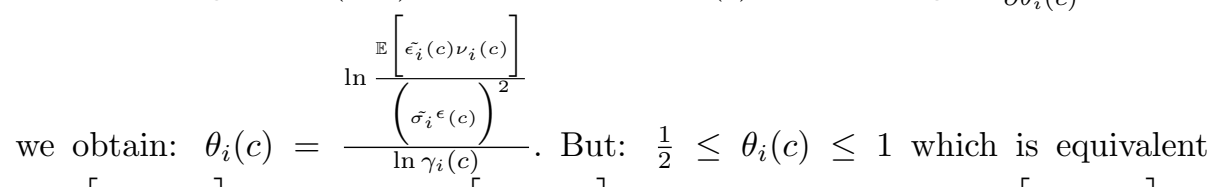
to $\frac{\mathbb{E}\left[\tilde{\epsilon_{i}}(c) \nu_{i}(c)\right]}{\left(\tilde{\sigma_{i}}{ }^{\epsilon}(c)\right)^{2}} \leq \gamma_{i}(c) \leq\left(\frac{\mathbb{E}\left[\tilde{\epsilon_{i}}(c) \nu_{i}(c)\right]}{\left(\tilde{\sigma_{i}}{ }^{\epsilon}(c)\right)^{2}}\right)^{2}$. Using the fact that $\frac{\mathbb{E}\left[\tilde{\epsilon_{i}}(c) \nu_{i}(c)\right]}{\left(\tilde{\sigma_{i}}{ }^{\epsilon}(c)\right)^{2}} \leq$ $\gamma_{i}(c)$, we check that $\theta_{i}(c)$ coincides with a minimum for $R \operatorname{Var}(i, T)$ indeed: $\frac{\partial^{2} R \operatorname{Var}(i, T)}{\partial \theta_{i}(c)^{2}}=2 \gamma_{i}(c)^{\theta_{i}(c)} \ln \left(\gamma_{i}(c)\right)^{2}\left[2\left(\tilde{\sigma}_{i}{ }^{\epsilon}(c)\right)^{2} \gamma_{i}(c)-\mathbb{E}\left[\tilde{\epsilon}_{i}(c) \nu_{i}(c)\right]\right]>0$.

MD 2: In Equations (8) and (9), $\hat{d}_{i}(c)$ and $\hat{w}_{i}(c)$ do not dependent on time. This implies that in the algorithms described in Subsection 4.3, the optimal positions are also independent on time; contrary to MD 1, where the optimal positions are functions of the day-ahead and real-time price ratio, which is time dependent. Using this observation, we infer that the day-ahead price is time independent; indeed: $p^{f}=\frac{\sum_{j}\left(\hat{d}_{j}-\hat{w}_{j}+r_{j}\right)+A^{f}}{B^{f}}$. 
In case of endogenous prices, market $i$ supplier investment takes the following form:

$$
\begin{aligned}
\mathcal{I}(i, T) & =\sum_{t}(\underbrace{\sum_{c \in \mathcal{C}_{i}}\left(\hat{d}_{i}(c)-\hat{w}_{i}(c)\right)}_{\hat{d}_{i}-\hat{w}_{i}}+r_{i}) p^{f}(t)+\sum_{t}[\underbrace{\sum_{c \in \mathcal{C}_{i}}\left(\tilde{\epsilon}_{i}(c, t) \gamma_{i}(c)^{\theta_{i}(c)}-\nu_{i}(c, t)\right)}_{\Delta_{i}(t)} \\
& \left.\left.-r_{i}\right)_{+} p_{i}^{0}(t)\right]+\sum_{c \in \mathcal{C}_{i}} \Xi\left(\gamma_{i}(c)\right)
\end{aligned}
$$

Compared to MD 1, we observe one additional difficulty: at the optimum $r_{i}$ relies on $\mathbf{r}_{-\mathbf{i}}$ and the bilateral trades rely on the decisions of the other markets.

Market $i$ supplier's risk is similar to the one derived for MD 1 :

$$
R \operatorname{Var}(i, T)=\operatorname{Var}\left(\mathcal{I}(i, T) \mid\left\{\Delta_{i}(t) \geq r_{i}\right\}_{t}\right)=\sigma_{\Delta_{i}}^{2} \sum_{t} p_{i}^{0}(t)^{2}
$$

Therefore, the same result holds about the optimal wind farm concentration. In MD 1 and MD 2, the MF is completely described by the set of equations summarized in the statement of Theorem 7

\subsection{In the $(\mathbb{E}[\mathcal{I}(i, T)], R \operatorname{Var}(i, T))$ plane}

The representation of the MF in the $(\mathbb{E}[\mathcal{I}(i, T)], R \operatorname{Var}(i, T))$ plane is not straightforward. Indeed, in case of endogenous prices, the optimal number of wind farms to construct in each cluster cannot be computed analytically. The optimal positions and the optimal bilateral trades can only be obtained algorithmically, as explained in Section 4.3 .

In each cluster $c \in \mathcal{C}_{i}$, the supplier can construct a fixed number of wind farms $\gamma_{i}(c)$. For each combination of $\left(\gamma_{i}(c)\right)_{i, c}$, we derive the optimal wind farm portfolio concentration $\left(\theta_{i}(c)\right)_{i, c}$ using Theorem 7. We substitute the resulting $\left(\gamma_{i}(c), \theta_{i}(c)\right)_{i, c}$ in $\left(\sigma_{\Delta_{i}}\right)_{i}$ as derived in Equation 10 and in the Nash Equilibrium in the positions and bilateral trades $\left(r_{i}^{*}, \Lambda_{i}^{*}\right)_{i}$ obtained through the algorithm detailed in Section 4.3.

We substitute $\left(r_{i}^{*}, \Lambda_{i}^{*}\right)_{i}$ and $\left(\Delta_{i}(t)\right)_{i}$ in the real time price:

$p_{i}^{0}(t)=\frac{A_{i}^{0}+\left(\Delta_{i}(t)-r_{i}^{*}\right)_{+}+\Lambda_{i}^{*}}{B_{i}^{0}}, \forall i=1, \ldots, N$ derived in Subsection 4.2 From this, we can infer the supplier's risk using Equation (12).

At the same time, we note that the expectation of market $i$ supplier invest- 
ment can be simplified to give:

$$
\begin{array}{r}
\mathbb{E}[\mathcal{I}(i, T)]=\frac{T}{B^{f}}\left(\hat{d}_{i}-\hat{w}_{i}+r_{i}\right)\left\{\sum_{j}\left(\hat{d}_{j}-\hat{w}_{j}+r_{j}\right)+A^{f}\right\}+\frac{T}{B_{i}^{0}}\left\{A_{i}^{0}\right. \\
\left.+\varphi_{i}\left(r_{i}, \mathbf{r}_{-\mathbf{i}}\right)\right\} \mathbb{E}\left[\left(\Delta_{i}(t)-r_{i}\right)_{+}\right]+\frac{T}{B_{i}^{0}} \mathbb{E}\left[\left(\Delta_{i}(t)-r_{i}\right)_{+}^{2}\right]+\sum_{c \in \mathcal{C}_{i}} \Xi\left(\gamma_{i}(c)\right)
\end{array}
$$

where, as detailed in Appendix: $\mathbb{E}\left[\left(\Delta_{i}(t)-r_{i}\right)_{+}\right]=\mathbb{E}\left[\left(\Delta_{i}(t)-r_{i}\right) \mid\left\{\Delta_{i}(t) \geq\right.\right.$ $\left.\left.r_{i}\right\}\right]=\frac{\sigma_{\Delta_{i}}}{\sqrt{2 \pi}} \exp \left(-\frac{r_{i}^{2}}{2 \sigma_{\Delta_{i}}^{2}}\right)-r_{i} \bar{F}_{\Delta_{i}}\left(r_{i}\right)$ and $\mathbb{E}\left[\left(\Delta_{i}(t)-r_{i}\right)_{+}^{2}\right]=\mathbb{E}\left[\left(\Delta_{i}(t)-r_{i}\right)^{2} \mid\left\{\Delta_{i}(t) \geq\right.\right.$ $\left.\left.r_{i}\right\}\right]=\frac{\sigma_{\Delta_{i}}^{2}}{\sqrt{\pi}} \Gamma\left(\frac{3}{2}\right) \Gamma_{i n c}\left(\frac{3}{2}, \frac{r_{i}^{2}}{2 \sigma_{\Delta_{i}}^{2}}\right)-\frac{\sigma_{\Delta_{i}}}{\sqrt{2 \pi}} \exp \left(-\frac{r_{i}^{2}}{2 \sigma_{\Delta_{i}}^{2}}\right)+r_{i}^{2}$ with $\Gamma(a)$ the Gamma function evaluated in $a \in \mathbb{R}_{+}$and $\Gamma_{i n c}(a, x)=\frac{1}{\Gamma(a)} \int_{x}^{+\infty} u^{a-1} \exp (-u) d u$ the Incomplete Gamma function with lower bound, evaluated in $a, x \in \mathbb{R}_{+}$.

\section{Numerical illustrations for three geographic demand markets: France, Germany and Bel- gium}

In the numerical illustrations, we consider three geographic demand markets: France, Germany and Belgium. Wind farm portfolio optimization is restricted to the French area, since our energy consumption data focus on this country.

The marginal cost parameters are based on Chao and Peck's six node toy network [5]: for France (Fr) we take $a_{F r}^{f}=42.5, a_{F r}^{0}=4250, b_{F r}^{f}=b_{F r}^{0}=0.025$; for Germany $(\mathrm{Ge})$ we take $a_{G e}^{f}=15, a_{G e}^{0}=1500, b_{G e}^{f}=b_{G e}^{0}=0.05$ and for Belgium (Be) $a_{B e}^{f}=10, a_{B e}^{0}=1000, b_{B e}^{f}=b_{B e}^{0}=0.05$.

The equivalent interconnection capacities are set so that: $\kappa_{B e, G e}=2(G W)$, $\kappa_{B e, F r}=6(G W)$ and $\kappa_{G e, F r}=5(G W)$.

\subsection{Description of the data and clustering of the geo- graphic demand markets}

For Germany, our database is made of time series of 75 sensors located all over Germany, providing one year wind speed measures (from 03/19/2013 until $03 / 18 / 2014$ ) with one measure per hour [32]. The exact GPS coordinates of the sensors are depicted by circles in Figure 2 (a). We use two Machine Learning techniques to partition the sensors based on the mean and variance of their wind speed time series: firstly, k-Means algorithm clusters data by separating samples in an a priori determined number of groups, minimizing a criterion known as the inertia of the groups. The optimal number of classes for the sensors (4) has been estimated a priori using an unsupervized clustering method known as affinity propagation [35. Secondly, one-class Support Vector Machine (SVM) can be used as a type of unsupervised learning algorithm, for novelty detection: given 
a set of samples, it will detect the soft boundary of that set so as to classify new points as belonging to that set or not. Both techniques give identical (or, at least, very close) classes, which are represented in Figure 2 (a) and (b). The convex hull of the sensor classes gives an approximation to the clusters geographic area for Germany.

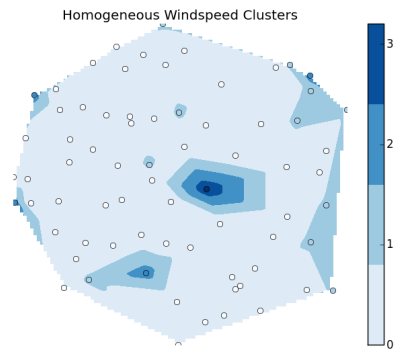

(a)

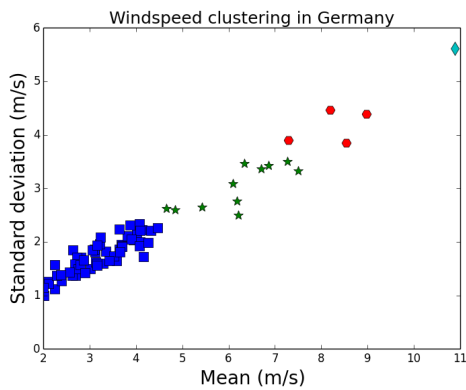

(b)

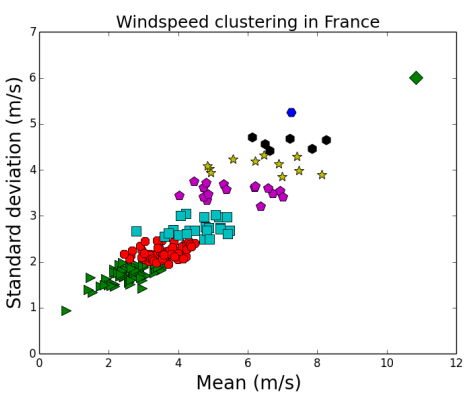

(c)

Figure 2: Partitioning Germany wind speed sensors, using a Machine Learning automatic partitioning algorithm (k-Means or SVM outlier detection) (a). The sensors' locations in GPS coordinates are represented in (a) by circles. In (b) and (c), the sensors are represented in the Mean-Variance plane depending on their wind speed time series mean and variance. We partition Germany and France's wind speed sensors in (b) and (c) respectively; each marker symbol being associated with a specific class.

For France and Belgium, we use data provided by the Météociel platform 33 for the year 2013. Each country is clusterized using one of the automatic partitioning tools, described above for Germany, applied on the historical wind speed measurements. We infer that France (resp. Belgium) can be partitioned in 8 (resp. 2) clusters, as represented in Figure2 (c) for France. The geographic coordinates of the cluster areas can be found in 3 . We associate an index with each French cluster area. The wind power production at the various points in the clusters are then inferred from the wind speed measurements using the Betz limit 10 Finally, over each cluster, the wind power productions are averaged.

\footnotetext{
${ }^{10}$ Source for Betz limit: http://www.wind-power-program.com/betz.htm
} 
The mean and standard deviations are then calculated on the basis of each cluster average time series of wind power production. We consider a constant average and standard deviation for wind power production throughout the time horizon.

Estimated wind production for Germany and Belgium is fixed so that: $\hat{w}_{G e}=$ $15(G W)$ and $\hat{w}_{B e}=10(G W)$. The forecast error difference standard deviations are set so that: $\sigma_{\Delta_{B e}}=\sigma_{\Delta_{G e}}=5(G W)$. For France, if 10 turbines were placed in each cluster, $\hat{w}_{F r}=4.97(G W)$ and the forecast error difference standard deviation would be: $\sigma_{\Delta_{F r}}=5.79(G W)$.

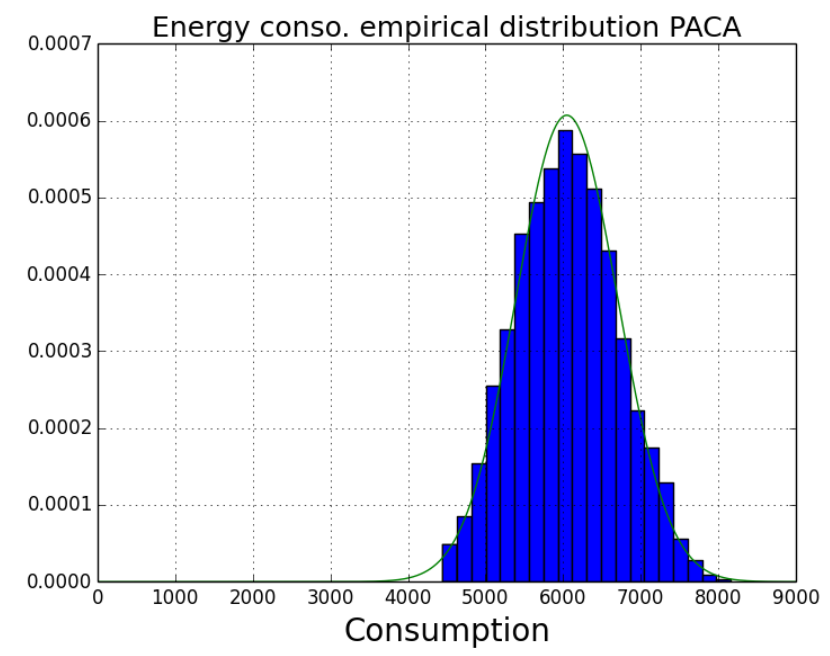

Figure 3: Energy consumption empirical distribution function (in $\mathrm{kWh}$ ) compared to the best fit Gaussian density function for PACA region.

For estimating the variance associated to the demand forecast, we use a data base containing one year (2013) power measurements (in kW) for each French region, with a granularity of one measure per 30 minutes [34]. The French regions are then mapped to the French clusters, as defined in RTE report [3]. Then, over each cluster, we take the average of these time series to obtain one value per day. In Figure 3, we compare the empirical distribution function of one French cluster (PACA region) energy consumption with the best fit Gaussian density function. This comparison validates our assumptions on demand forecasting, captured in Equation (8).

For France, the end users' total demand is estimated by: $\hat{d}_{F r}=56.173(G W)$; for Germany and Belgium we fix: $\hat{d}_{G e}=\hat{d}_{B e}=40(G W)$. 


\begin{tabular}{|l|l|l|l|l|l|l|l|l|}
\hline $\begin{array}{l}\text { Clust. } \\
\text { ind. }\end{array}$ & $\mathbf{1}$ & $\mathbf{2}$ & $\mathbf{3}$ & $\mathbf{4}$ & $\mathbf{5}$ & $\mathbf{6}$ & $\mathbf{7}$ & $\mathbf{8}$ \\
\hline $\begin{array}{l}\text { Mean } \\
\text { conso. }\end{array}$ & 19.890 & 6.043 & 2.480 & 2.947 & 9.104 & 4.875 & 3.736 & 7.098 \\
\hline $\begin{array}{l}\text { Conso. } 5.048 \\
\text { stand. } \\
\text { dev. }\end{array}$ & 1.196 & 0.496 & 0.654 & 2.010 & 1.032 & 0.953 & 1.874 \\
\hline
\end{tabular}

Table 1: Mean consumption (GW) and consumption standard deviation per French cluster (GW).

\subsection{Optimal forward positions and Markowitz Frontier representations for France}

In Figure 4 (a), we plot market $i$ optimal position for the two tiered market with exogenous prices (MD 1) described in Section 3 as a function of the exogenous price ratio $\frac{p_{F r}^{0}}{p^{f}}$ and of the forecast error standard deviation $\sigma_{\Delta_{F r}}$. In Figure 4(b), we plot the optimal position for the two tiered market with endogenous prices (MD 2) and positions described in Section 4 as a function of the wind forecast $\hat{w}_{F r}$ and of the forecast error standard deviation $\sigma_{\Delta_{F r}}$. The endogenous prices corresponding to the interval of variation of $\hat{w}_{i}$ and $\sigma_{\Delta_{i}}$ are such that $p^{f}$ belongs to the interval $[0 ; 95 ; 1.30]$ and $p^{0}$ to the interval $[3.52 ; 4.16]$. For the exogenous prices, we choose $p^{0}=4$ which is close to the center of the interval of variation of the real-time endogenous price. The day-ahead endogenous price then varies in the interval $[0.15 ; 1.35]$. The endogenous and exogenous prices are now in the same range of values. We observe that in case of exogenous prices, $\frac{p^{0}}{p^{f}}$ weakly impacts the optimal position whereas $\sigma_{\Delta_{i}}$ clearly makes the position increase; in case of endogenous prices, both $\hat{w}_{i}$ and $\sigma_{\Delta_{i}}$ make the position increase. Reciprocally, the increase of the position makes $\frac{p^{0}}{p^{f}}$ decrease in case of endogenous prices whereas it has only a threshold effect in case of exogenous prices.

In Figure 5, we plot the MF for the wind farm portfolio over each French cluster as a function of the number of turbines and of the concentration of the wind farms over the cluster. These plots are issued from the theoretical relation derived in Theorem 7

We assume that the supplier can construct 0,60 or 120 turbines over each cluster leading to $3^{8}$ combinations for France. This choice of numerical values is justified by the fact that largest US wind farms nowadays have around 600 turbines 11 and by coming down on the scale of France. Assuming that a wind farm counts on the average 60 turbines, the supplier has the choice between constructing 0,1 or 2 wind farms per cluster. We observe in Figure 6 that the value of $\xi$ and the form of the investment cost (i.e., linear, quadratic, etc.)

\footnotetext{
${ }^{11}$ For example, the Roscoe wind farm in Roscoe, Texas, is one of the world's largest capacity
} wind farms with 634 turbines and a total installed capacity of $781.5 \mathrm{MW}$. 


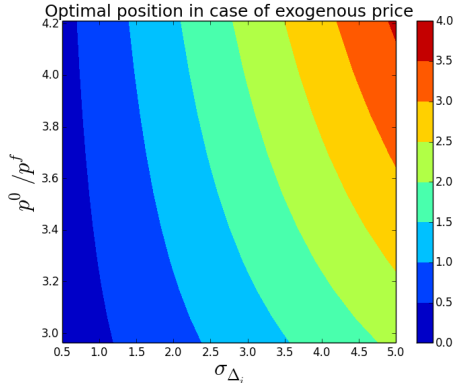

(a)

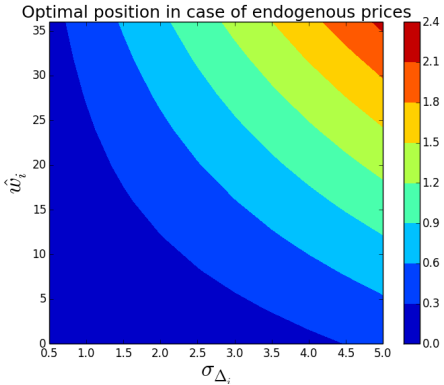

(b)

Figure 4: In (a) (resp. (b)), we represent the optimal position $r_{i}$ in case of exogenous prices (resp. endogenous prices) as a function of the forecast error difference standard deviation $\sigma_{\Delta_{i}}$ and of the exogenous price ratio $\left(\frac{p^{0}}{p^{f}}\right)$ (resp. of the wind forecast $\hat{w}_{i}$ ).

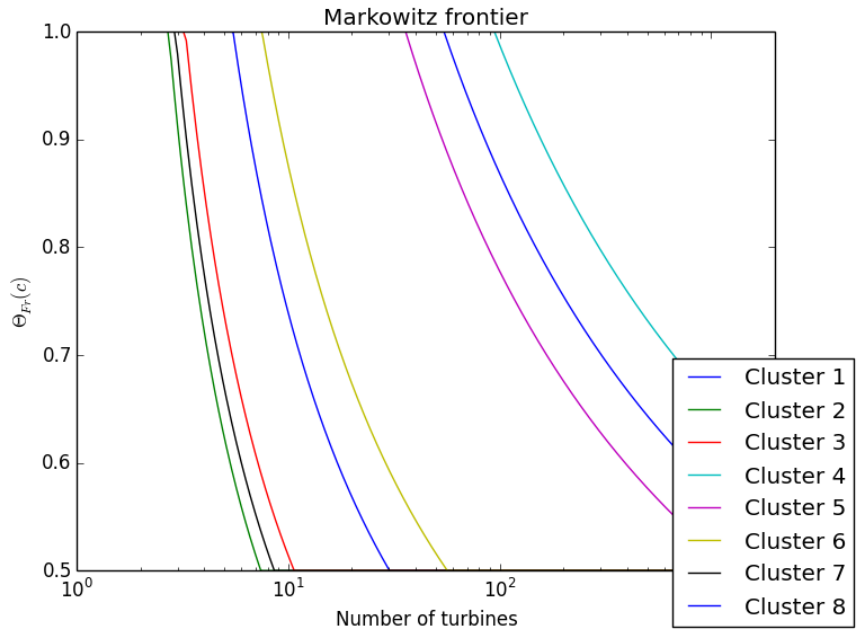

Figure 5: Over each French cluster, the Markowitz Frontier for the wind farm portfolio is represented as a function of the number of turbines and of the concentration between the wind farms over the cluster. Each of the 8 French clusters is characterized by a specific color. 
deeply influence the MF shape.
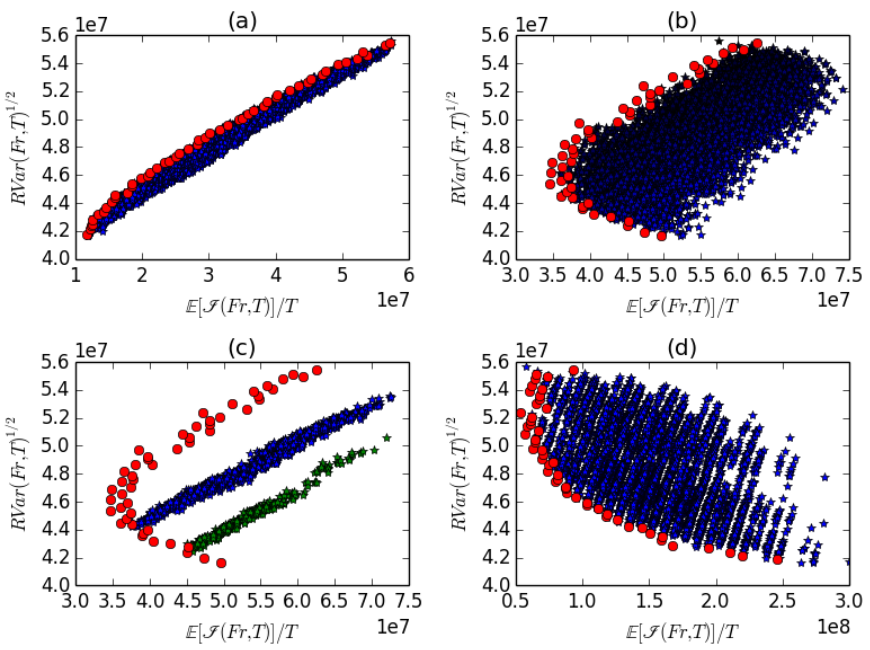

Figure 6: Markowitz Frontier (MF) in red in the $\left(\frac{\mathbb{E}[\mathcal{I}(F r, T)]}{T}, \operatorname{RV} \operatorname{ar}(F r, T)^{1 / 2}\right)$ plane. In (a), we represent all the portfolio combinations and the $M F$ for $\frac{\xi}{T}=1$ and a linear cost of construction. In (b), we plot all the portfolio combinations and the MF for $\frac{\xi}{T}=900$ and a linear cost of construction. In (c), the same setting holds but we select exclusively the portfolios associated with two construction costs: $\sum_{c \in \mathcal{C}_{F r}} \frac{\Xi\left(\gamma_{F r}(c)\right)}{T}=$ $24.10^{3}$ and $36.10^{3}$. In (d), we plot the portfolio combinations and the MF for $\frac{\xi}{T}=1$ and a quadratic cost of construction.

\section{Conclusion}

We considered a stylized network model representing a certain number of geographic demand markets and assumed rational expectation of the agents. The originality of our work relies on the introduction of competition among the geographic demand markets and of our choice to model the agent interactions as a Signaling Game. In each geographic demand market, supplier optimizes selfishly its day-ahead position which acts as a signal for the generators which optimize their bilateral trades with the other markets. We proved analytically that firstly, there exist conditions guaranteeing the existence and the uniqueness of a Nash Equilibrium for less or exactly three geographic demand markets and secondly, that the set of efficient wind farm portfolios can be expressed as a function of the number of constructed wind farms and of their concentration independently of the Market Design. Finally, we simulated, on a real case study, the contour of the Markowitz Frontier in the expected investment-risk plane. 
The linearity of the cost functions, the inelasticity of demand and day-ahead Market Coupling are necessary assumptions in our analysis. The relaxation of these assumptions is an interesting direction of future research but will lead us to the limits of analytical tractability.

\section{Appendix: Proof of Proposition 5}

By substitution of the day-ahead and real-time prices at equilibrium obtained in Lemmas 1 and 2 in market $i$ expected procurement cost, we obtain:

$$
U_{i}=q_{i}^{f} \frac{\sum_{j} q_{j}^{f}+A^{f}}{B^{f}}+\mathbb{E}\left[\frac{\left(q_{i}^{0}\right)^{2}}{B_{i}^{0}}+\frac{A_{i}^{0}+\sum_{j \neq i} \lambda_{i \rightarrow j}^{0}}{B_{i}^{0}} q_{i}^{0}-\tilde{p_{i}^{0}} \tilde{q}_{i}^{0}\right]
$$

Using Proposition 3 and the fact that $\mathbb{E}\left[q_{i}^{0}\right]=\mathbb{E}\left[\left(\Delta_{i}-r_{i}\right)_{+}\right]=\mathbb{E}\left[\left(\Delta_{i}-\right.\right.$ $\left.\left.r_{i}\right) \mid\left\{\Delta_{i} \geq r_{i}\right\}\right]$ and $\mathbb{E}\left[\tilde{q}_{i}^{0}\right]=\mathbb{E}\left[\left(\Delta_{i}-r_{i}\right)_{-}\right]=\mathbb{E}\left[\left(\Delta_{i}-r_{i}\right) \mid\left\{\Delta_{i} \leq r_{i}\right\}\right]$, we obtain:

$$
\begin{aligned}
\frac{\partial U_{i}}{\partial r_{i}} & =\frac{\sum_{j} q_{j}^{f}+A^{f}}{B^{f}}+\frac{q_{i}^{f}}{B^{f}}+\frac{\partial}{\partial r_{i}}\left(\frac{1}{B_{i}^{0}} \mathbb{E}\left[\left(\Delta_{i}-r_{i}\right)^{2} \mid\left\{\Delta_{i} \geq r_{i}\right\}\right]\right) \\
& +\frac{A_{i}^{0}+\sum_{j \neq i} \lambda_{i \rightarrow j}^{0}}{B_{i}^{0}} \frac{\partial}{\partial r_{i}} \mathbb{E}\left[\left(\Delta_{i}-r_{i}\right) \mid\left\{\Delta_{i} \geq r_{i}\right\}\right] \\
& +b_{i}^{0}\left(\frac{1}{B^{f} b_{i}^{f}}-1\right) \mathbb{E}\left[\left(\Delta_{i}-r_{i}\right) \mid\left\{\Delta_{i} \geq r_{i}\right\}\right]-\tilde{p}_{i}^{0} \frac{\partial}{\partial r_{i}} \mathbb{E}\left[\left(\Delta_{i}-r_{i}\right) \mid\left\{\Delta_{i} \leq r_{i}\right\}\right]
\end{aligned}
$$

Since the forecast error differences $\Delta_{i}$ are distributed according to Gaussian distribution functions centered in 0 and of standard deviation $\sigma_{\Delta_{i}}$, it is possible to express the first and second derivatives of $\left(\Delta_{i}-r_{i}\right)$ and $\left(\Delta_{i}-r_{i}\right)^{2}$ conditionally to the event $\left\{\Delta_{i} \geq r_{i}\right\}$ as functions of the Incomplete Gamma function which enables us to derive the following closed forms:

$$
\begin{aligned}
\mathbb{E}\left[\left(\Delta_{i}-r_{i}\right) \mid\left\{\Delta_{i} \geq r_{i}\right\}\right] & =\int_{r_{i}}^{+\infty} \Delta f_{\Delta_{i}}(\Delta) d \Delta-r_{i} \int_{r_{i}}^{+\infty} f_{\Delta_{i}}(\Delta) d \Delta \\
& =\frac{\sigma_{\Delta_{i}}}{\sqrt{2 \pi}} \exp \left(-\frac{r_{i}^{2}}{2 \sigma_{\Delta_{i}}^{2}}\right)-r_{i} \bar{F}_{\Delta_{i}}\left(r_{i}\right) \\
\frac{\partial}{\partial r_{i}} \mathbb{E}\left[\left(\Delta_{i}-r_{i}\right) \mid\left\{\Delta_{i} \geq r_{i}\right\}\right] & =-\bar{F}_{\Delta_{i}}\left(r_{i}\right) \\
\frac{\partial^{2}}{\partial r_{i}^{2}} \mathbb{E}\left[\left(\Delta_{i}-r_{i}\right) \mid\left\{\Delta_{i} \geq r_{i}\right\}\right] & =f_{\Delta_{i}}\left(r_{i}\right)
\end{aligned}
$$

and

$$
\begin{aligned}
\mathbb{E}\left[\left(\Delta_{i}-r_{i}\right)^{2} \mid\left\{\Delta_{i} \geq r_{i}\right\}\right] & =\int_{r_{i}}^{+\infty} \Delta^{2} f_{\Delta_{i}}(\Delta) d \Delta-2 r_{i} \int_{r_{i}}^{+\infty} \Delta f_{\Delta_{i}}(\Delta) d \Delta \\
& +r_{i}^{2} \int_{r_{i}}^{+\infty} f_{\Delta_{i}}(\Delta) d \Delta
\end{aligned}
$$




$$
\begin{aligned}
\frac{\partial}{\partial r_{i}} \mathbb{E}\left[\left(\Delta_{i}-r_{i}\right)^{2} \mid\left\{\Delta_{i} \geq r_{i}\right\}\right] & =2\left[r_{i} \int_{r_{i}}^{+\infty} f_{\Delta_{i}}(\Delta) d \Delta-\int_{r_{i}}^{+\infty} \Delta f_{\Delta_{i}}(\Delta) d \Delta\right] \\
\frac{\partial^{2}}{\partial r_{i}^{2}} \mathbb{E}\left[\left(\Delta_{i}-r_{i}\right)^{2} \mid\left\{\Delta_{i} \geq r_{i}\right\}\right] & =2 \bar{F}_{\Delta_{i}}\left(r_{i}\right)
\end{aligned}
$$

Using the same principle, we obtain:

$$
\frac{\partial}{\partial r_{i}} \mathbb{E}\left[\left(\Delta_{i}-r_{i}\right) \mid\left\{\Delta_{i} \leq r_{i}\right\}\right]=F_{\Delta_{i}}\left(r_{i}\right)
$$

Using Equations (13), 14 and (15), we obtain:

$$
\frac{\partial^{2} U_{i}}{\partial r_{i}^{2}}=\frac{2}{B^{f}}+b_{i}^{0}\left(3-\frac{1}{B^{f} b_{i}^{f}}\right) \bar{F}_{\Delta_{i}}\left(r_{i}\right)+\frac{\sum_{j \neq i} \lambda_{i \rightarrow j}^{0}-\left(\tilde{p_{i}^{0}} B_{i}^{0}-A_{i}^{0}\right)}{B_{i}^{0}} f_{\Delta_{i}}\left(r_{i}\right)
$$

Since $\bar{F}_{\Delta_{i}}\left(r_{i}\right)>0$ and $f_{\Delta_{i}}\left(r_{i}\right)>0$ for any $r_{i} \in \mathbb{R}, B^{f} b_{i}^{f}>1$ by definition and $B^{f}>0, B_{i}^{0}>0$, the sign of $\frac{\partial^{2} U_{i}}{\partial r_{i}^{2}}$ for $r_{i} \in[0 ;+\infty[$ depends on the sign of $\frac{\sum_{j \neq i} \lambda_{i \rightarrow j}^{0}-\left(\tilde{p_{i}^{0}} B_{i}^{0}-A_{i}^{0}\right)}{B_{i}^{0}}$. Two cases are possible:

Case 1: $\frac{\sum_{j \neq i} \lambda_{i \rightarrow j}^{0}-\left(\tilde{p_{i}^{0}} B_{i}^{0}-A_{i}^{0}\right)}{B_{i}^{0}} \geq 0 \Leftrightarrow \sum_{j \neq i} \lambda_{i \rightarrow j}^{0} \geq\left(\tilde{p_{i}^{0}} B_{i}^{0}-A_{i}^{0}\right)$

This first case corresponds to the case where the quantity of imports is not too high compared to the quantity of exports, for market $i$. In this first case, we infer that $\frac{\partial^{2} U_{i}}{\partial r_{i}^{2}}>0, \forall r_{i} \geq 0$. Hence $U_{i}$ is convex in $r_{i} \geq 0$. Therefore, there exists a unique $r_{i} \geq 0$ minimizing $U_{i}$.

Case 2: $\frac{\sum_{j \neq i} \lambda_{i \rightarrow j}^{0}-\left(\tilde{p_{i}^{0}} B_{i}^{0}-A_{i}^{0}\right)}{B_{i}^{0}}<0 \Leftrightarrow \sum_{j \neq i} \lambda_{i \rightarrow j}^{0}<\left(\tilde{p_{i}^{0}} B_{i}^{0}-A_{i}^{0}\right)$

This second case corresponds to the case where the quantity of imports is high compared to the quantity of exports, for geographic market $i$.

Derivating three times $U_{i}$ with respect to $r_{i}$, we obtain:

$$
\begin{aligned}
\frac{\partial^{3} U_{i}}{\partial r_{i}^{3}} & =-b_{i}^{0}\left(3-\frac{1}{B^{f} b_{i}^{f}}\right) f_{\Delta_{i}}\left(r_{i}\right)-\frac{\left(A_{i}^{0}-B_{i}^{0} \tilde{p}_{i}^{0}\right)+\sum_{j \neq i} \lambda_{i \rightarrow j}^{0}}{B_{i}^{0}} \frac{r_{i}}{\sigma_{\Delta_{i}}^{2}} f_{\Delta_{i}}\left(r_{i}\right) \\
& =-\frac{f_{\Delta_{i}}\left(r_{i}\right)}{B_{i}^{0}} \frac{1}{\sigma_{\Delta_{i}}^{2}}\left[\left(3-\frac{1}{B^{f} b_{i}^{f}}\right) \sigma_{\Delta_{i}}^{2}+r_{i}\left(\left(A_{i}^{0}-B_{i}^{0} \tilde{p}_{i}^{0}\right)+\sum_{j \neq i} \lambda_{i \rightarrow j}^{0}\right)\right]
\end{aligned}
$$

using the fact that $\frac{d f_{\Delta_{i}}\left(r_{i}\right)}{d r_{i}}=-\frac{r_{i}}{\sigma_{\Delta_{i}}^{2}} f_{\Delta_{i}}\left(r_{i}\right), \forall r_{i} \in \mathbb{R}$ since $\Delta_{i}$ is distributed according to a Gaussian distribution function centered in 0 and of standard deviation $\sigma_{\Delta_{i}}$. Then: $\frac{\partial^{3} U_{i}}{\partial r_{i}^{3}}=0 \Leftrightarrow r_{i}=-\frac{\left(3-\frac{1}{B^{f} b_{i}^{f}}\right) \sigma_{\Delta_{i}}^{2}}{\left(A_{i}^{0}-B_{i}^{0} \tilde{p}_{i}^{0}\right)+\sum_{j \neq i} \lambda_{i \rightarrow j}^{0}}$. 
We set: $r_{i}^{0} \triangleq-\frac{\left(3-\frac{1}{B^{f} b_{i}^{f}}\right) \sigma_{\Delta_{i}}^{2}}{\left(A_{i}^{0}-B_{i}^{0} \tilde{p}_{i}^{0}\right)+\sum_{j \neq i} \lambda_{i \rightarrow j}^{0}}$. Then, we note that:

- If $r_{i}<r_{i}^{0}$ then $r_{i}<-\frac{\left(3-\frac{1}{B^{f} b_{i}^{f}}\right) \sigma_{\Delta_{i}}^{2}}{\left(A_{i}^{0}-B_{i}^{0} \tilde{p}_{i}^{0}\right)+\sum_{j \neq i} \lambda_{i \rightarrow j}^{0}} \Leftrightarrow\left(3-\frac{1}{B^{f} b_{i}^{f}}\right) \sigma_{\Delta_{i}}^{2}+r_{i}\left(\left(A_{i}^{0}-\right.\right.$ $\left.\left.B_{i}^{0} \tilde{p}_{i}^{0}\right)+\sum_{j \neq i} \lambda_{i \rightarrow j}^{0}\right)>0$. This implies in turn that $\frac{\partial^{3} U_{i}}{\partial r_{i}^{3}}<0$.

- Identically, if $r_{i}>r_{i}^{0}$ then $\frac{\partial^{3} U_{i}}{\partial r_{i}^{3}}>0$.

Both of these observations imply that $\frac{\partial^{2} U_{i}}{\partial r_{i}^{2}}$ is decreasing on $\left[0 ; r_{i}^{0}[\right.$ and increasing on $\left[r_{i}^{0} ;+\infty\left[\right.\right.$. Furthermore, the number of points where $\frac{\partial^{2} U_{i}}{\partial r_{i}^{2}}=0$ depend on the value of $\left.\frac{\partial^{2} U_{i}}{\partial r_{i}^{2}}\right|_{r_{i}=r_{i}^{0}}$.

Case $2(a):\left.\frac{\partial^{2} U_{i}}{\partial r_{i}^{2}}\right|_{r_{i}=r_{i}^{0}}>0$

Then $\frac{\partial^{2} U_{i}}{\partial r_{i}^{2}}>0, \forall r_{i} \geq 0$. This implies that $U_{i}$ is convex on $\mathbb{R}_{+}$. Therefore it admits a unique minimum on $[0 ;+\infty[$.

Case $2(b):\left.\frac{\partial^{2} U_{i}}{\partial r_{i}^{2}}\right|_{r_{i}=r_{i}^{0}}<0$

Then two sub-cases should be distinguished depending on the sign of $\left.\frac{\partial^{2} U_{i}}{\partial r_{i}^{2}}\right|_{r_{i}=0}$.

Case $2(b)(i):\left.\frac{\partial^{2} U_{i}}{\partial r_{i}^{2}}\right|_{r_{i}=0}<0$ There exists a unique $r_{i}^{1} \in\left[r_{i}^{0} ;+\infty[\right.$ such that $\frac{\partial^{2} U_{i}}{\partial r_{i}^{2}}<0$ on $\left[0 ; r_{i}^{1}\right.$ [ and $\frac{\partial^{2} U_{i}}{\partial r_{i}^{2}} \geq 0$ on $\left[r_{i}^{1} ;+\infty[\right.$. This implies that $\frac{\partial U_{i}}{\partial r_{i}}$ is decreasing on $\left[0 ; r_{i}^{1}\left[\right.\right.$ and increasing on $\left[r_{i}^{1} ;+\infty[\right.$. Hence $\frac{\partial U_{i}}{\partial r_{i}}=0$ in 0,1 or 2 points for $r_{i} \geq 0$. Then: either $\left.\frac{\partial U_{i}}{\partial r_{i}}\right|_{r_{i}=r_{i}^{1}}>0$ which implies that $U_{i}$ is strictly increasing on $\mathbb{R}_{+}$reaching its minimum in $r_{i}=0$; or there exists a point $r_{i}^{*} \in\left[r_{i}^{1} ;+\infty[\right.$ such that $\left.\frac{\partial U_{i}}{\partial r_{i}}\right|_{r_{i}=r_{i}^{*}}=0$. Since $\frac{\partial^{2} U_{i}}{\partial r_{i}^{2}}>0$ on $\left[r_{i}^{1} ;+\infty\left[\right.\right.$ only, this implies that $r_{i}^{*}$ is the unique minimum of $U_{i}$ on $\mathbb{R}_{+}$.

Case $2(b)(i i):\left.\frac{\partial^{2} U_{i}}{\partial r_{i}^{2}}\right|_{r_{i}=0}>0$ There exist $0 \leq r_{i}^{2} \leq r_{i}^{0}$ and $r_{i}^{0} \leq r_{i}^{3}$ such that $\frac{\partial^{2} U_{i}}{\partial r_{i}^{2}}>0$ on $\left[0 ; r_{i}^{2}\left[,<0\right.\right.$ on $\left[r_{i}^{2} ; r_{i}^{3}\left[\right.\right.$ and $>0$ on $\left[r_{i}^{3} ;+\infty[\right.$.

If $\left.\frac{\partial U_{i}}{\partial r_{i}}\right|_{r_{i}=0} \geq 0$ then there exists a unique $r_{i}^{*} \in\left[r_{i}^{3} ;+\infty[\right.$ such that $\left.\frac{\partial U_{i}}{\partial r_{i}}\right|_{r_{i}=r_{i}^{*}}=0$ and $\left.\frac{\partial^{2} U_{i}}{\partial r_{i}^{2}}\right|_{r_{i}=r_{i}^{*}}>0$. In this case, $r_{i}^{*}$ is the unique minimum of $U_{i}$ over $\mathbb{R}_{+}$.

Otherwise i.e., if $\left.\frac{\partial U_{i}}{\partial r_{i}}\right|_{r_{i}=0}<0$ then: either $\left.\frac{\partial U_{i}}{\partial r_{i}}\right|_{r_{i}=r_{i}^{2}}<0$ in which case $U_{i}$ admits a unique minimum over $\mathbb{R}_{+}$belonging to $\left[r_{i}^{3} ;+\infty\left[\right.\right.$; or $\left.\frac{\partial U_{i}}{\partial r_{i}}\right|_{r_{i}=r_{i}^{2}} \geq 0$ in which case $U_{i}$ admits two minima over $\mathbb{R}^{+}$, the first one in $\left[0 ; r_{i}^{2}\right.$ [ and the second one in $\left[r_{i}^{3} ;+\infty[\right.$. 
The case $\left.\frac{\partial^{2} U_{i}}{\partial r_{i}^{2}}\right|_{r_{i}=0}>0$ should be avoided since it might give rise to a large number of equilibria $\left(2^{N}\right)$ for Program 3. Therefore, in the case where $\sum_{j \neq i} \lambda_{i \rightarrow j}^{0}<\left(\tilde{p_{i}^{0}} B_{i}^{0}-A_{i}^{0}\right)$, it might be reasonable to impose some conditions on the problem parameters so that Case $2(b)(i i)$ is avoided. In other words:

$$
\begin{aligned}
\left.\frac{\partial^{2} U_{i}}{\partial r_{i}^{2}}\right|_{r_{i}=0} & =\frac{2}{B^{f}}+\frac{1}{2 B_{i}^{0}}\left(3-\frac{1}{B^{f} b_{i}^{f}}\right)+\frac{\sum_{j \neq i} \lambda_{i \rightarrow j}^{0}-\left(\tilde{p_{i}^{0}} B_{i}^{0}-A_{i}^{0}\right)}{B_{i}^{0}} f_{\Delta_{i}}(0)<0 \\
& \Leftrightarrow f_{\Delta_{i}}(0)>-\frac{\frac{2 B_{i}^{0}}{B^{f}}+\frac{1}{2}\left(3-\frac{1}{B^{f} b_{i}^{f}}\right)}{\sum_{j \neq i} \lambda_{i \rightarrow j}^{0}-\left(\tilde{\left.p_{i}^{0} B_{i}^{0}-A_{i}^{0}\right)}\right.} \\
& \Leftrightarrow \sigma_{\Delta_{i}}<-\frac{1}{\sqrt{2 \pi}}\left[\frac{\sum_{j \neq i} \lambda_{i \rightarrow j}^{0}-\left(\tilde{p_{i}^{0}} B_{i}^{0}-A_{i}^{0}\right)}{\frac{2 B_{i}^{0}}{B^{f}}+\frac{1}{2}\left(3-\frac{1}{B^{f} b_{i}^{f}}\right)}\right]
\end{aligned}
$$

Therefore, to avoid Case $2(b)$ (ii), the standard-deviation related to the knowledge of $\Delta_{i}$ should be smaller than $\frac{1}{\sqrt{2 \pi}}\left[\frac{\sum_{j \neq i} \lambda_{i \rightarrow j}^{0}-\left({\tilde{p_{i}^{0}}}_{i}^{0}-A_{i}^{0}\right)}{\frac{1}{2}\left(\frac{1}{B^{f} b_{i}^{f}}-3\right)-\frac{2 B_{i}^{0}}{B^{f}}}\right]$.

\section{References}

[1] Baringo L., Conejo A. J., Strategic Wind Power Investment, IEEE Transactions on Power Systems, vol.29, pages 1250 - 1260, 2014

[2] Bertsekas D. P., Nonlinear programming, Athena Scientific, 1999

[3] Biégala N., Coulondre J.-M., Report on Fixed Characteristics Assessment of Intermediate Scaled Model, RTE Optimate D1.2 Deliverable, 2011

[4] Borenstein S., Bushnell J., Stoft S., The competitive effects of transmission capacity in a deregulated electricity industry, RAND Journal of Economics, vol.31, pages $294-325,2000$

[5] Chao H. P., Peck S. C., Reliability management in competitive electricity markets, Journal of Regulatory Economics, vol.14, pages 198 - 200, 1998

[6] Daxhelet O., Smeers Y., The EU regulation on cross-border trade of electricity: A two-stage equilibrium model, European Journal of Operational Research, vol.181, pages $1396-1412,2007$

[7] Girard R., Laquaine K., G. Kariniotakis, Assessment of wind power predictability as a decision factor in the investment phase of wind farms, Applied Energy, vol.16, pages $51-63,2013$

[8] Glachant J.-M., Ruester S., The EU internal electricity market: Done forever?, Utilities Policy, vol.30, pages 1 - 7, 2014 
[9] Green R., Staffell I., How Large Should A Portfolio of Wind Farms Be?, in proceedings of The Economics of Energy Markets, TSE, 2013

[10] Hagspiel S., Jägemann C., Lindenberger D., Brown T., Cherevatskiy S., Tröster E., Cost-Optimal Power System Extension under Flow-Based Market Coupling, Energy, vol.66, pages 654-666, 2014

[11] Hutcheon N., Bialek J. W., Updated and validated power flow model of the continental European transmission network, in proceedings of IEEE PowerTech, 2013

[12] Kogan J., Introduction to Clustering Large and High-Dimensional Data, Cambridge University Press, 2007

[13] Kunz F., Improving congestion management: how to facilitate the integration of renewable generation in germany?, The Energy journal, vol.34, pages $55-78,2013$

[14] Le Cadre H., Bedo J.-S., Distributed Learning in the Smart Grid: A Learning Game Approach, Preprint Online in HAL https://hal. archives-ouvertes.fr/hal-00740893v7/document, 2014

[15] Le Cadre H., Didier M., Quantifying the Impact of Unpredictable Generation on Market Coupling, Preprint Online in $H A L$ https://hal.archives-ouvertes.fr/file/index/docid/1038387/ filename/OPTE_623_Version_1_coupling_journalV4.pdf, 2014

[16] Le Cadre H., Mercier D., Is Energy Storage an Economic Opportunity for the Eco-Neighborhood, NETNOMICS: Economic Research and Electronic Networking, vol.13, pages $191-216,2012$

[17] Markowitz H., Portfolio Selection, The Journal of Finance, vol.7, pages $77--91,1952$

[18] Marling H., Emanuelsson S., The Markowitz Portfolio Theory, Survey Online http://www.math.chalmers.se/, 2012

[19] Martin S., Smeers Y., Aguado J. A., A Stochastic Two-Settlement Equilibrium Model for Electricity Markets with Wind Generation, IEEE Transactions on Power Systems, vol.30, pages 233 - -245, 2014

[20] Morales J. M., Zugno M., Pineda S., Pinson P., Electricity Market Clearing with Improved Scheduling of Stochastic Production, European Journal of Operational Research, vol.235, pages $765--774,2014$

[21] Nair J., Adlakha S., Wierman A., Energy Procurement Strategies in the Presence of Intermittent Sources, ACM SIGMETRICS Performance Evaluation Review, vol.42, pages $85-97,2014$ 
[22] Neuhoff K., Hobbs B., Newbery D., Congestion Management in European Power Networks, Deutsches Institut für Wirtschafsforschung Discussion Paper, 2011

[23] Newbery D., Strbac G., Viehoff I., The benefits of integrating European electricity markets, EPRG Working Paper $n^{o}$ 1504, Cambridge Working Paper in Economics, 2012

[24] Oggioni G., Murphy F. H., Smeers Y., Evaluating the impacts of priority dispatch in the European electricity market, Energy Economics, vol.42, pages $183-200,2014$

[25] Papavasiliou A., Oren S., Multi-Area Stochastic Unit Commitment for High Wind Penetration in a Transmission Constrained Network, Operations Research, vol.61, pages $578-592,2013$

[26] Percebois J., Les Mécanismes de Soutien aux Energies Renouvelables, Cahier de recherche CREDEN $n^{\circ} 14.03 .107,2014$

[27] Pritchard G., Zakeri G., Philpott A., A Single-settlement, energy-only electric power market for unpredictable and intermittent participants, Operations Research, vol.58, pages 1210 - 1219, 2010

[28] Rösner S., Compléments de rémuération: La vente directe en Allemagne, 2014 https://enr-ee.com/fr/nouvelles/article/150/ presentation-de-lofaenr-sur-la-vente-directe-delectricite- $\backslash$ renouvelable-en-allemagne/

[29] Sinden G., Characteristics of the UK wind resource: Long-term patterns and relationship to electricity demand, Energy Policy, vol.35, pages $112-$ 127,2007

[30] Smeers Y., Oggioni G., Allevi E., Schaible S., Generalized Nash Equilibrium and Market Coupling in the European Power System, Networks and Spatial Economics, vol.12, pages 503-560, 2012

[31] Sobel J., Signaling Games, Encyclopedia of Complexity and Systems Science, pages $8125-8139,2009$

[32] Deutscher Wetterdienst, https://werdis.dwd.de/

[33] Météociel platform, http://meteociel.fr/obs/pays.php.

[34] French regional consumption data, http://www.rte-france.com/fr/ developpement-durable/eco2mix/telechargement-de-donnees

[35] Scikit-learn, Python toolbox for Machine Learning, http: //scikit-learn.org/ 
[36] Survey on Ancillary Services Procurement and Balancing Market Design, March 2014, https://www.entsoe.eu/fileadmin/user_ upload/_library/publications/entsoe/ENTSO-E_2013_Survey_on_ AS_Procurement_and_EBM_design.pdf

[37] ELIA: The CIPU contract, a set framework for taking part in the high-voltage grid management, July 2014, http://www.elia. be/ /media/files/Elia/Products-and-services/ProductSheets/ S-Ondersteuning-net/S5_F_CIPU_08_07.pdf

[38] ELIA: Tertiary production reserve, a solution to major imbalances and congestion, 2014, http://www.elia.be/ /media/files/Elia/ Products-and-services/ProductSheets/S-Ondersteuning-net/S3_F_ RES_TERT_PROD.pdf

[39] ELIA: Règles de Fonctionnement du marché relatif à la compensation des déséquilibres quart-horaires - Entrée en vigueur partiellement en 2014 et intégralement à partir du 1-er janvier 2015, May 2014, http://www.elia.be/ /media/files/Elia/Products-and-services/ Balancing/Balancing_Rules_06052014.pdf

[40] Potential cross-border balancing cooperation between the Belgian, Dutch and German electricity Transmission System Operators, October 2014, http://www.tennet.eu/nl/fileadmin/downloads/About_ Tennet/Publications/Technical_Publications/balancing/141008_ Final_report.pdf

[41] Euphemia Public Description, November 2013, http: //www.npspot.com/globalassets/Download\%20Center/PCR/ Euphemia-public-description_Nov2013.pdf 